\title{
The structure of electrospray beams in vacuum
}

\author{
MANUEL GAMERO-CASTAÑO \\ Department of Mechanical and Aerospace Engineering, University of California, \\ Irvine, CA 92717, USA
}

(Received 12 July 2007 and in revised form 24 February 2008)

Electrospray atomization of liquids in the cone-jet mode generates narrow droplet distributions with average diameters as small as a few nanometres. This ability is important for technologies such as colloid thrusters, nanoparticle generation and ion beam processes, and the optimization of these applications requires an understanding of the physics and structure of the associated beams. This paper presents a detailed experimental characterization of electrosprays in vacuum and formulates an analytical model of the beam. A key feature of our model is the use of a simplified expression for the electric field induced by the space charge. This simplification leads to a time-independent Eulerian formulation compatible with an analytical solution, in contrast to the direct simulation of a multitude of droplets which must be simultaneously tracked to account for Coulombic interactions. We find that the beams open up in an initial region relatively insensitive to the external electrodes, a process dominated by the electric repulsion between droplets and the initial droplet inertia. Although the external electric field modifies the trajectories of the droplets downstream of this initial region, the effect is moderate in our typical electrospray source and the analytical solution in the space charge region explains well the far-field beam structure observed experimentally. We also describe a numerical scheme that implements the full effect of the external electric field and provides a more accurate solution.

\section{Introduction}

Electrohydrodynamic spraying in the cone-jet mode (Cloupeau \& Prunet-Foch 1989 ) is the only technique known to atomize dielectric liquids into similar submicrometric droplets. The axial component of the electric field induced by a Taylor cone accelerates the charged jet emanating from its tip, and reduces its initial diameter (Gañán-Calvo 1997). Disturbances on the jet surface are initially damped by the fast acceleration of the jet, but as the electric field weakens and the acceleration slows, perturbation modes with the appropriate wavelength grow into droplets. The breakup of a cylindrical jet is a phenomenon accurately described by the theory of capillary instability, which explains the observed narrow distribution of droplet diameters on the basis of the exponential growth of axisymmetric perturbation modes, together with the existence of a critical wavelength of maximum growth speed (Chandrasekhar 1981). The droplets emitted by cone-jets can be smaller than those produced by other techniques relaying on a jet breakup, e.g. direct pressure atomizers or the remarkable pneumatic atomizer of Gañán-Calvo (1998), because the initial diameter of the electrospray jet can be made as small as a few nanometres by choosing/controlling the properties of the liquid (especially its electrical conductivity, Fernández de la Mora \& Loscertales 1994), and because the strong and stabilizing electrical force further thins 
(a)

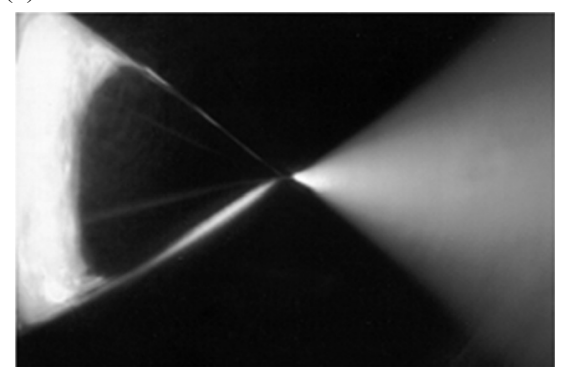

(b)

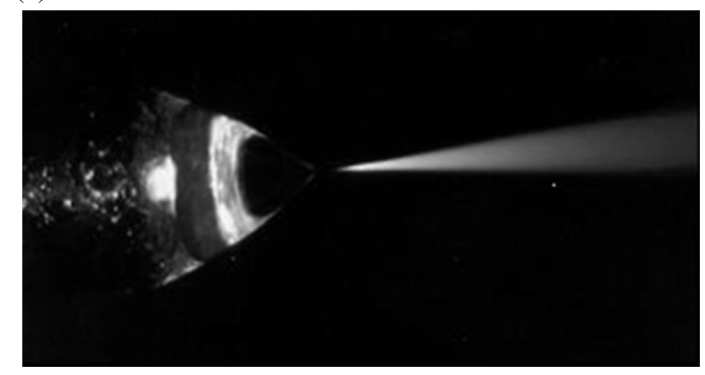

FIGURE 1. Electrosprays at atmospheric pressure $(a)$ and vacuum $(b)$. The image in vacuum shows from left to right: the capillary emitter (approximately $1 \mathrm{~mm}$ outer diameter), the Taylor cone, and the beam of droplets. The atmospheric drag reduces the velocity of the droplets, and increases the space charge density and the spread of the beam.

the jet. Ion field evaporation (Gamero-Castaño 2002), which changes the physics of the cone-jet and seems to preclude the formation of a jet, sets a minimum of the order of a few nanometres for the initial diameter of electrospray droplets (Fernández de la Mora 2007).

Technologies using electrospray atomization are improved by, or require, a detailed knowledge of the structure of the associated beams. Fernández de la Mora (1992) has solved the particular case of a droplet velocity field proportional to the electric field, i.e. the inertialess droplet case typical of highly conducting liquids at atmospheric pressure. Among other results, Fernández de la Mora's solution explains the observed departure of liquid cone angles from the spray-free value of $49^{\circ}$ computed by Taylor (1964), and predicts the droplet density as a function of position. This information is important to optimize, for example, the initial sampling stage of an electrospray mass spectrometer, which is by far the most important application of electrosprays (Fenn et al. 1989). The present paper deals with the opposite case in which droplet inertia is significant, and only the electrostatic force is important. The ideal example is that of an electrospray in vacuum, a case of particular interest for colloid thrusters and ion beam applications. Figure 1 shows the clear difference between electrospray beams held at atmospheric pressure and under vacuum. The drag on the droplets at atmospheric pressure reduces their velocities, increases the relative importance of repulsion between droplets, and augments the spread of the beam.

The goal of this paper is to find the functional dependency between the beam structure of a cone-jet in vacuum and the properties of the droplets. The paper is organized as follows: first, we provide a detailed characterization of five electrosprays of a propylene carbonate solution. Section 3 introduces a mathematical model for the trajectories of droplets. The analysis of the equations shows that the electric repulsion between droplets is the dominant force in a small region near the emission point, an observation that leads to the simplification of the equations and to an approximate analytical solution valid inside the space charge region. After this, we compute a more accurate solution by numerically integrating the trajectories between the emission point and the extractor plane. The importance of the experimental characterization, which provides the input parameters required by the beam model and validates the solution, is made apparent throughout $\S 3$. Finally, $\S 4$ summarizes the results. 


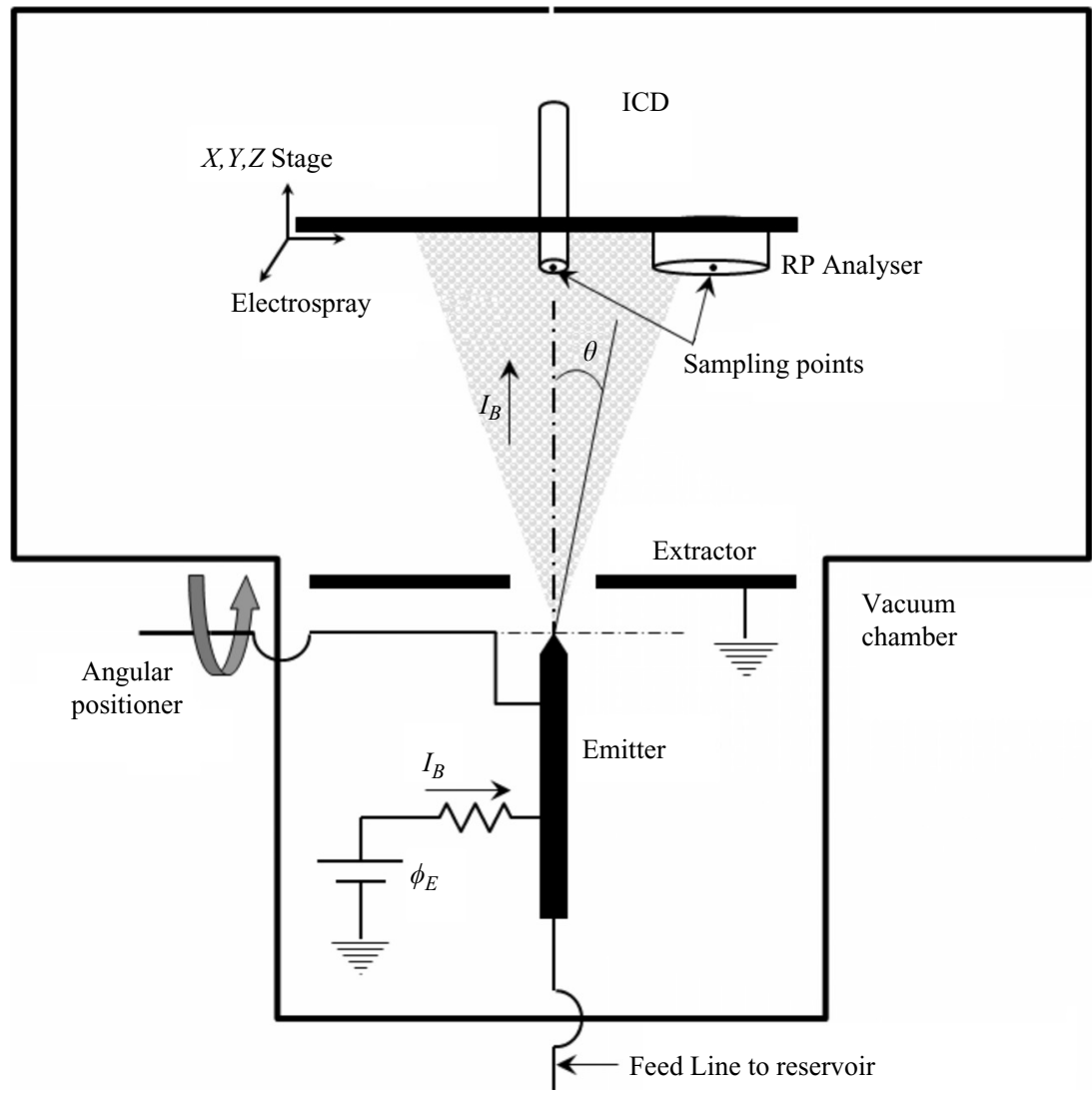

FIGURE 2. Experimental set-up. The electrospray source is inside a vacuum chamber. Its beam of charged droplets is characterized with a retarding potential analyser and an induction charge detector. The polar angle $\theta$ is defined by the emission point and the axis of the beam.

\section{Experimental characterization}

\subsection{Experimental arrangement and beam profiles}

Figure 2 shows the experimental set-up. The electrosprays are generated inside a vacuum chamber, at a pressure in the $10^{-5}-10^{-6}$ Torr range. The emitter is a stainless steel capillary tube separated from an extracting electrode by a $3.17 \mathrm{~mm}$ gap. The outer and inner diameters of the emitter are 0.23 and $0.11 \mathrm{~mm}$, while the extractor orifice has a diameter of $3.95 \mathrm{~mm}$. The opposite end of the capillary is inserted in a reservoir with the liquid and placed outside the vacuum chamber. The pressure inside the reservoir is adjusted to drive the desired flow rate. When an appropriate voltage difference $\phi_{E}$ is applied between the emitter and extractor electrodes (typically $1950 \mathrm{~V}$ in these experiments), the liquid at the emitter tip shapes into a Taylor cone and breaks into droplets at its apex. The resulting beam of droplets passes through the extractor orifice and enters a larger area of the vacuum chamber where it is characterized by a retarding potential analyser (RPA) with a sampling orifice of $4.97 \mathrm{~mm}^{2}$, and by an induction charge detector (ICD, Gamero-Castaño 2007) that senses individual droplets. The RPA can also measure the flux of charge through its sampling orifice when no retarding potential barrier is applied, rendering in this mode a profile of the beam current. Downstream of the extractor, the droplet trajectories 


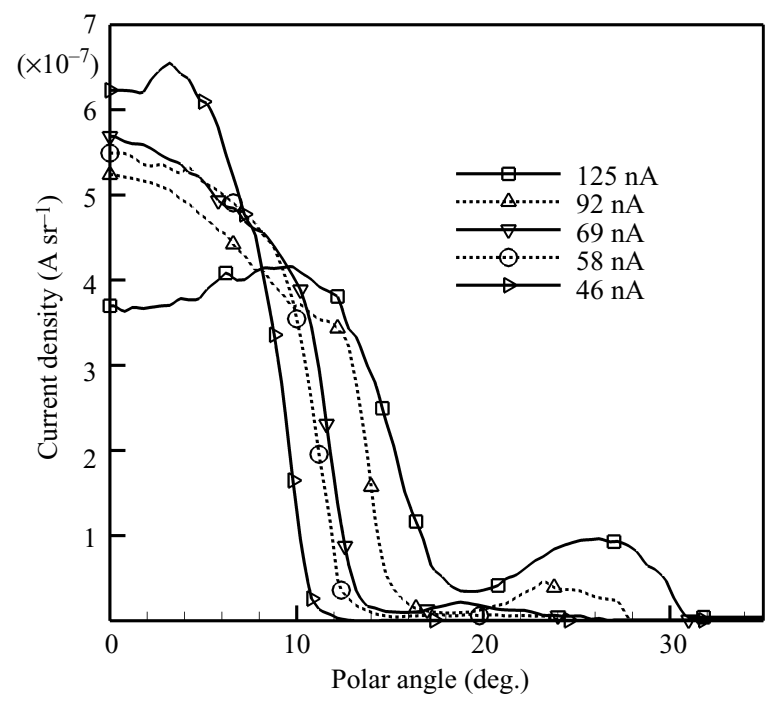

FIGURE 3. Current per solid angle as a function of polar angle (beam profiles). The five electrospray currents show a pattern of increasing satellite droplet generation for increasing beam current.

are approximately straight lines originating from the emitter tip; the polar angle $\theta$ used throughout the paper is defined by the emission point (the origin of a spherical coordinate system) and the beam axis (polar axis). Both the RPA and the ICD are mounted on an $X, Y, Z$ positioning stage to sample any point in the beam. In addition, the electrospray source can be rotated around an axis intersecting the tip of the emitter, which lets the ICD study droplets at any polar angle (the axis of the ICD cannot be rotated).

Our emitter-extractor geometry is typical of electrospray applications that require shielding of the emission region from external electric fields. The orifice in the extracting electrode allows the outflow of the electrospray beam to generate thrust in a colloid thruster, deposit droplets on a surface, analyse the properties of the droplets, etc. The extractor orifice increases the radial component of the electric field induced by the electrodes. The larger radial field slightly increases the exit angle of the trajectories of droplets.

The liquid electrosprayed is a $0.033 \%$ (vol) solution of 1-ethyl-3-methylimidazolium bis(trifluoromethylsulfonyl) imide (McEwen et al. 1999) in propylene carbonate. The ionic liquid provides the free charge required by the electrospraying process. Propylene carbonate was chosen because of its low vapour pressure (required for vacuum operation), and high dielectric constant (to dissolve and dissociate high concentrations of electrolytes). The density $\rho$, viscosity $\mu$, surface tension $\gamma$, dielectric constant $\varepsilon$, and an estimate of the vapour pressure of pure propylene carbonate are $1200 \mathrm{~kg} \mathrm{~m}^{-3}$, $0.00276 \mathrm{~kg} \mathrm{~s}^{-1} \mathrm{~m}^{-1}, 0.0419 \mathrm{~N} \mathrm{~m}^{-1}, 64.9$ and $2.9 \mathrm{~Pa}\left(20^{\circ} \mathrm{C}\right.$, Riddick, Bunger \& Sakano 1986). We will use these values for the mixture because of the low solute concentration.

Figure 3 shows the beam profiles (current per unit solid angle as a function of polar angle) of five electrosprays with beam currents of 46, 58, 69, 92 and $125 \mathrm{nA}$. The sprays become broader for increasing current. The profile for $46 \mathrm{nA}$ resembles a rectangle function with an edge sharply falling to zero current density. The $58 \mathrm{nA}$ profile has a similar shape, except for a small current tail extending beyond $14^{\circ}$. As the electrospray current increases, the area under this tail becomes larger; it separates from the central 
region of the beam, and the overall profile becomes the superposition of two fairly disconnected coaxial beams. These profile measurements, together with the RPA and ICD data described in the following paragraphs, show that the inner and outer beams are formed by two distinct populations of droplets: main and satellite. The inner beam carries main droplets, while the outer beam is made of satellite droplets. The main droplets of a given electrospray have larger diameters, mass to charge ratios, and retarding potentials than their satellite counterparts (Gamero-Castaño \& Hruby 2002).

Ashgriz \& Mashayek (1995) describe the generation of satellite droplets in axisymmetric breakups. They find that satellites originate when the breakup point in the sinusoidal disturbance shifts from its neck towards its swell, an event happening for large enough values of the Reynolds number, $R e=(1 / \mu)\left(\rho \gamma R_{J}\right)^{1 / 2}\left(R_{J}\right.$ is the radius of the jet). At large $R e$, the fluid in the ligament between two consecutive main droplets detaches from them and becomes a smaller satellite droplet. Conversely, at low $R e$, the breakup point occurs at the neck of the disturbance and satellite droplets do not develop. Our charged jets display a pattern consistent with this picture: the jet radius scales linearly with the beam current (Gamero-Castaño \& Hruby 2002), making the Reynolds number an increasing function of the latter; at the lowest beam currents, and therefore at the lowest Reynolds numbers, there are no satellite droplets; however, as the beam current and the Reynolds number increase, a critical point is reached that triggers the formation of satellite droplets, and increasing numbers of them are generated thereafter.

\subsection{Measurement of the retarding potential of droplets}

The retarding potential of a droplet $\phi_{R P}$, defined as the sum of its kinetic and potential energy divided by the charge

$$
\phi_{R P}=\frac{1}{2 \xi} \mathrm{v}^{2}(x+\phi(x)
$$

is a constant of the droplet in an electrostatic field (Enloe \& Shell 1992). $\xi$ stands for the charge to mass ratio of the droplet. The electric field in the spray fluctuates, especially near the emission point, because of the stochastic generation and motion of different droplets. However, we will show in $\S 2.4$ that the random contribution to the retarding potential of droplets caused by the Coulombic interaction between them is small, and we will assume that the retarding potentials remain constant. Figure 4 shows retarding potential cumulative distributions for the $92 \mathrm{nA}$ electrospray at different polar angles. The electric potential of the emitter is $1950 \mathrm{~V}$. An error function is fitted to each data set to eliminate noise, and to illustrate that the density distributions $f\left(\phi_{R P}\right)$ are Gaussian-like. The mean retarding potentials of main droplets slowly decrease for increasing polar angle, especially near the axis. The standard deviations also decrease for increasing polar angle. The means of the satellite droplet distributions are considerably lower, they decrease with increasing polar angle, and the distributions are narrower.

Figure 5 shows the emitter potential minus the mean retarding potential, $\phi_{E}-$ $\left\langle\phi_{R P}\right\rangle$, and the standard deviation $\operatorname{STD}\left(\phi_{R P}\right)$, as functions of the polar angle. The trends described above for the $92 \mathrm{nA}$ case are common to all beams: initially, the mean retarding potential remains approximately constant, or slowly decreases, as the polar angle increases; the mean retarding potential decreases faster when the polar angle approaches the edge of the inner beam; and the mean retarding potentials for satellite droplets are significantly lower than for main droplets. The 
(a)

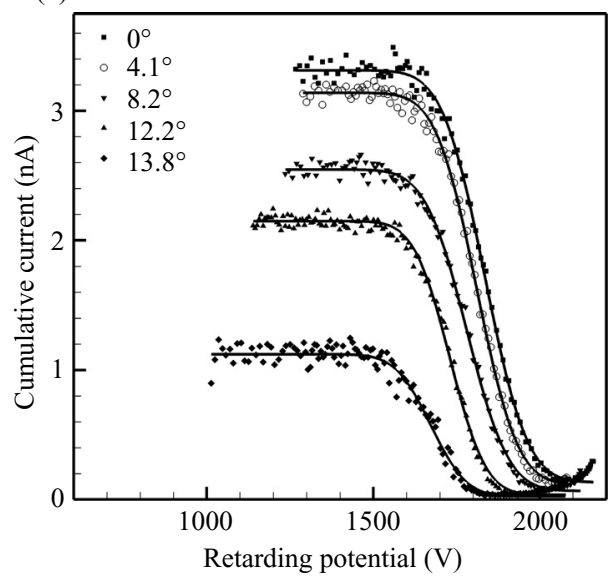

(b)

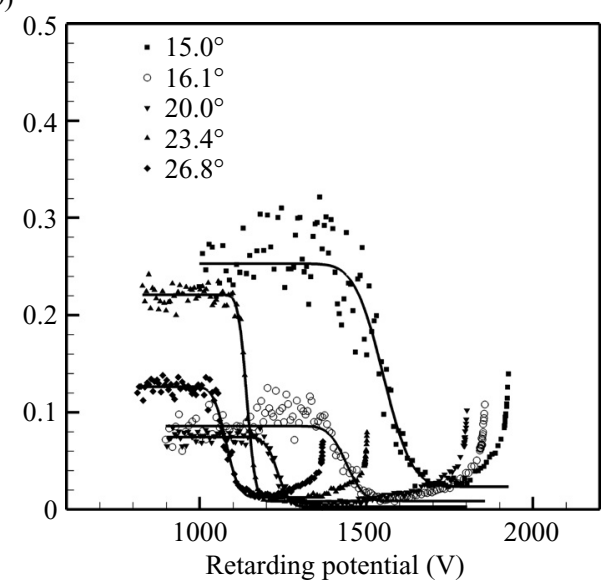

FIGURE 4. Retarding potential cumulative distributions at different polar angles $\left(I_{B}=92 \mathrm{nA}\right)$.
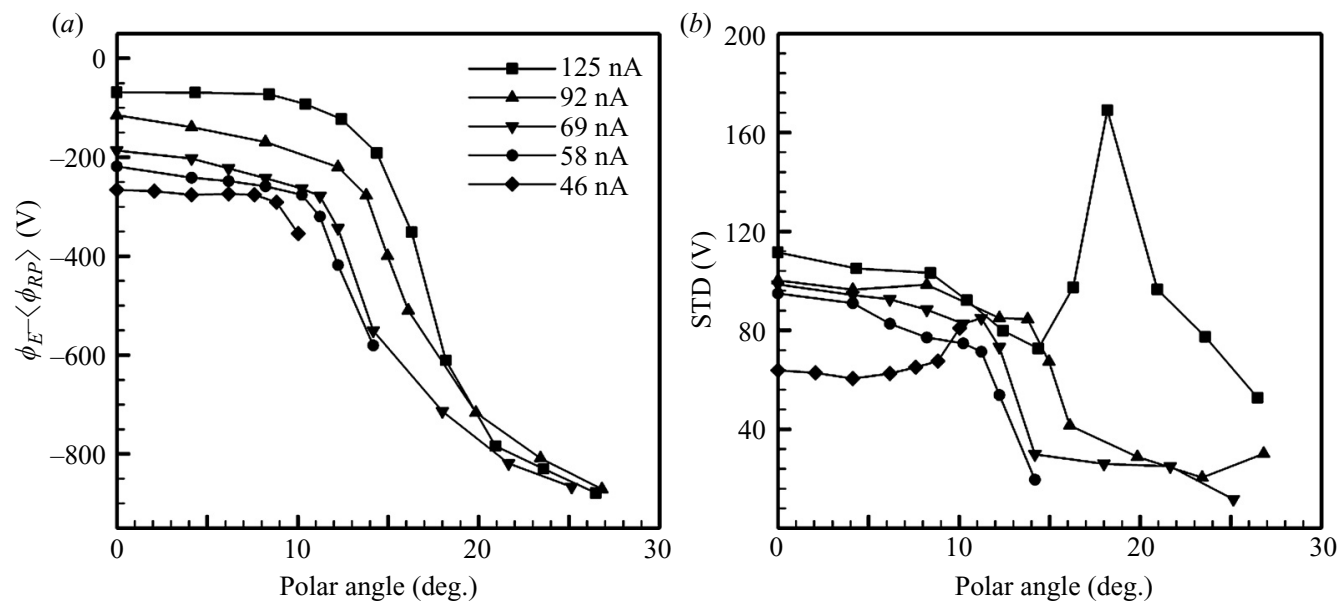

FIGURE 5. Emitter potential minus mean retarding potential, and standard deviation of the retarding potential distribution, at different polar angles.

dependence of the standard deviation on the polar angle displays a similar pattern: the distributions become slightly narrower as the polar angle increases; and for a given electrospray, the distributions of satellite droplets are narrower than those of main droplets. The last observation does not hold for the $125 \mathrm{nA}$ electrospray, which has a spike around $18.5^{\circ}$. This is due to the larger region of overlap between the satellite and main droplets, as shown in figure 3 . We do not differentiate between the two droplet populations when computing the standard deviation. Thus, if a location with a mixture of main and satellite droplets is sampled, the dissimilar retarding potentials result in a large standard deviation for the mixture.

\subsection{Measurement of the diameter and the charge of droplets}

The ICD measures the charge and time of flight TOF of a droplet (Shelton, Hendricks \& Wuerker 1960). The time of flight, combined with the retarding potential (the laboratory ground is the reference for the electric potential, while the potential 


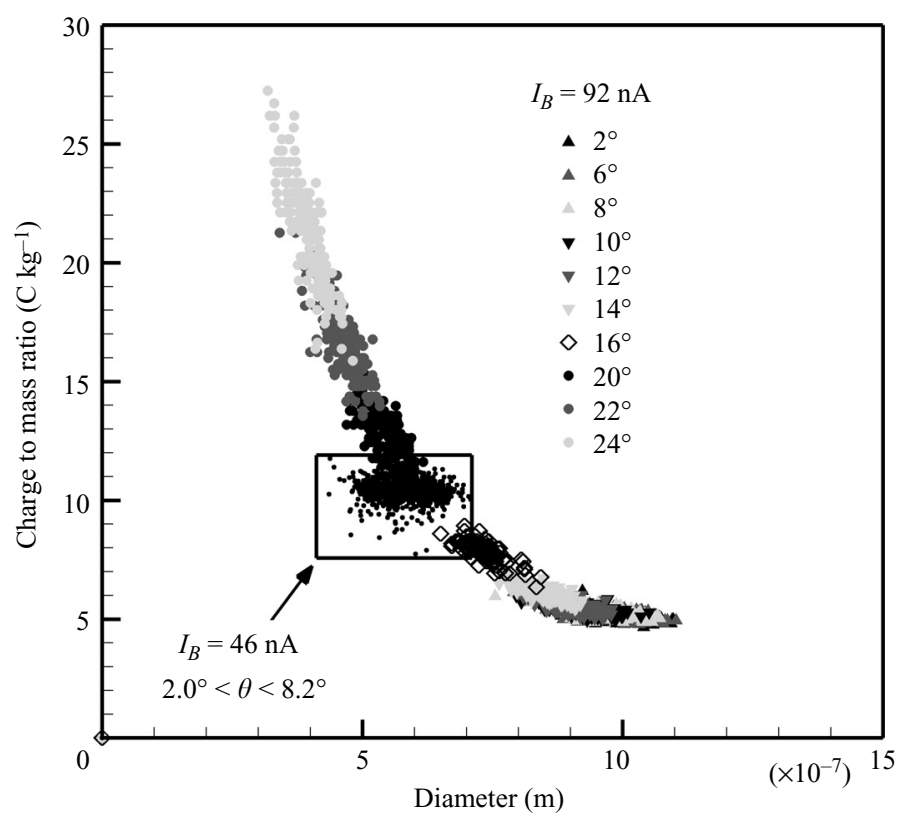

Figure 6. Charge to mass ratio versus diameter of individual droplets $\left(I_{B}=92 \mathrm{nA}, 46 \mathrm{nA}\right)$.

difference between the electrodes of the ICD and the laboratory ground is negligible), yield the charge to mass ratio:

$$
\xi=\frac{1}{2 \phi_{R P}}\left(\frac{L_{T O F}}{T O F}\right)^{2} .
$$

$L_{T O F}$ is the distance covered by the droplet during the time of flight measurement, $49.5 \mathrm{~mm}$ in our ICD (Gamero-Castaño 2007). The mass is computed with the measured charge and charge to mass ratio. The diameter of the droplet is obtained from its mass and the density of propylene carbonate.

The RPA measures the retarding potential distribution of droplets crossing a small sampling area, rather than the retarding potential of individual droplets. Because these distributions are relatively narrow, we will assign the mean retarding potential for a given polar angle to all droplets sampled at that angle, and use this value to compute their charge to mass ratios. Figure 6 plots the charge to mass ratio versus diameter of individual droplets for the $92 \mathrm{nA}$ electrospray, grouped by polar angles. Approximately 200 droplets are sampled at each polar angle. Figure 6 also shows the droplets of the $46 \mathrm{nA}$ beam measured throughout its angular range. We think that the use of an average retarding potential instead of each droplet's value is a major reason for the variability of $\xi$ at fixed diameter. Nevertheless, the spread is small and it is appropriate to define a function $\xi(D)$. This functional dependence is much weaker than the $\xi \propto 1 / D^{2}$ law consistent with an equipotential breakup (the jet evolving into a droplet is regarded as a perfect conductor), the hypothesis commonly used to model the effects of electrification on capillary jet breakup (López-Herrera \& Gañán-Calvo 2004; Collins, Harris \& Basaran 2007).

Figure 7 shows the mean diameter and the mean charge to mass ratio versus polar angle. The vertical bars across the symbols display the standard deviations of each angular sample (the standard deviation is half the bar length). The charge 

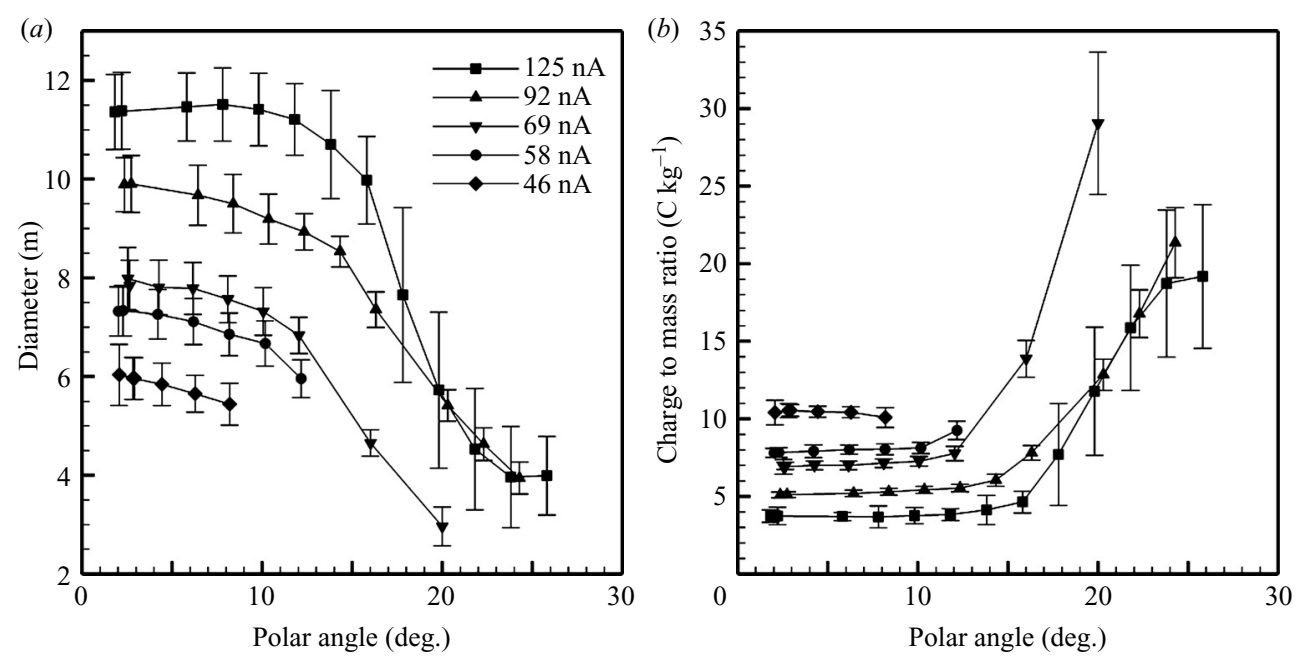

Figure 7. Mean diameter and mean charge to mass ratio of droplets at different polar angles. Vertical bars display the standard deviation of the distributions.

to mass ratio of main droplets is approximately constant for a given beam current, regardless of the polar angle. $\xi$ increases slightly along the edge of the main drop beam, and becomes substantially larger for satellite droplets. The charge to mass ratio of satellites increases substantially with the polar angle. The distributions of main droplet diameters are relatively broad at each polar angle, and the mean diameter decreases for increasing $\theta$. At polar angles before the edge of the main droplet beam, the decreasing trend is too weak to overcome the considerable variability, resulting in a barely noticeable angular separation of main droplets by diameter. The $125 \mathrm{nA}$ beam, having main droplet diameter distributions that are virtually independent of $\theta$, is an extreme example of this phenomenon. The mean of the diameter distributions decreases markedly along the edge of the main droplet beam, and at still larger polar angles the satellite droplets have much smaller diameters. When comparing different electrosprays, the diameters and mass to charge ratios increase with the beam current.

\subsection{Estimation of the initial velocity and potential of droplets. Line of point charges approximation}

The velocity of the droplets and the electrical potential at the jet breakup are initial and boundary conditions for the beam model. To estimate these parameters we will assume that the jet breaks at a constant axial location $\boldsymbol{x}_{J}$, generating droplets with a narrow distribution of velocities and electrical potentials (the means of these distributions must be similar to the velocity and potential of the jet at the breakup point, $v_{J}$ and $\left.\phi_{J}\right)$. We then average the retarding potential at $\boldsymbol{x}_{J}$ of all droplets sampled at a given polar angle

$$
\left\langle\phi_{R P}\right\rangle_{\theta}=\left\langle\frac{1}{2 \xi} \boldsymbol{v}^{2}\left(\boldsymbol{x}_{J}\right)+\phi\left(\boldsymbol{x}_{J}\right)\right\rangle_{\theta} \cong\left\langle\frac{1}{2 \xi}\right\rangle_{\theta} \boldsymbol{v}_{J}^{2}+\phi_{J} .
$$

Figure 8 plots $\left\langle\phi_{R P}\right\rangle_{\theta}$ versus $\langle 1 / 2 \xi\rangle_{\theta}$ for the 5 electrosprays, together with linear fittings. According to (3), the slope of the fitting is the square of the jet velocity at the breakup, while its electrical potential is given by the interception with the $y$-axis. Figure 8 shows that the data for the three larger currents do follow a linear 


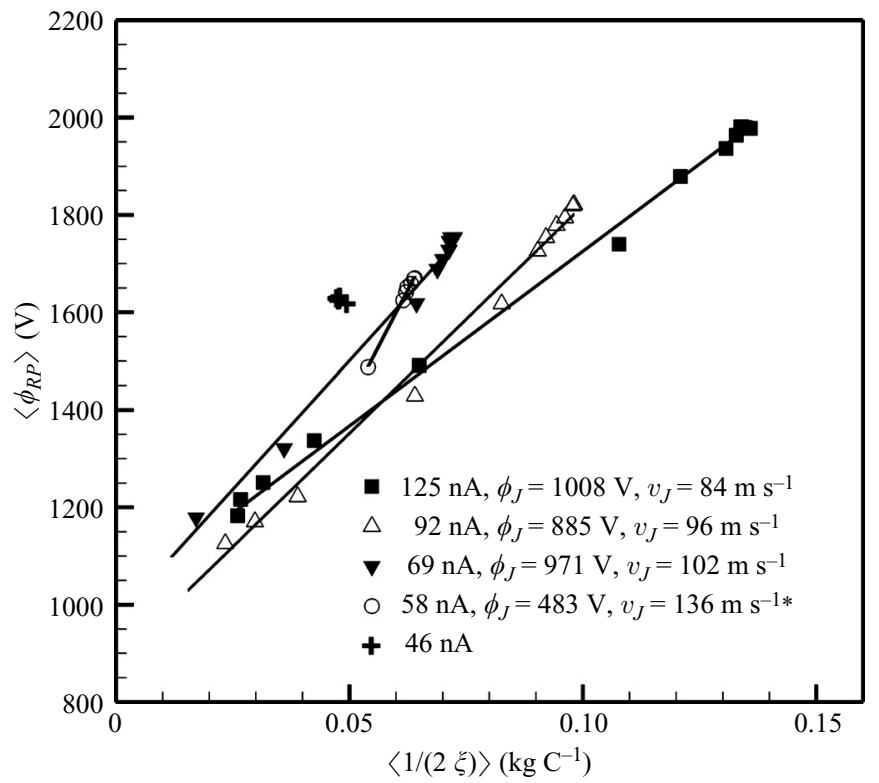

FIGURE 8 . The potential and velocity of the jet at its breakup point are estimated by taking advantage of the distributions of charge to mass ratio and retarding potential typical of electrospray droplets.

$\begin{array}{ccccccc}I_{B}(\mathrm{nA}) & Q\left(\mu \mathrm{g} \mathrm{s}^{-1}\right) & \phi_{E}(\mathrm{~V}) & \phi_{J}(\mathrm{~V}) & v_{J}\left(\mathrm{~m} \mathrm{~s}^{-1}\right) & D_{J}(\mu \mathrm{m}) & \langle D\rangle(\mu \mathrm{m}) \\ 125 & 25.2 & 2050 & 1008 & 84 & 0.56 & 1.13 \\ 92 & 14.4 & 1950 & 885 & 96 & 0.40 & 0.91 \\ 69 & 8.74 & 1950 & 971 & 103 & 0.30 & 0.72 \\ 58 & 7.13 & 1900 & 483^{*} & 136^{*} & 0.23^{*} & 0.69 \\ 46 & 4.19 & 1900 & & & & 0.56\end{array}$

* These estimates are inaccurate because of the narrow range of charge to mass ratios available for the $58 \mathrm{nA}$ electrospray.

TABLE 1. Relevant parameters of the electrosprays: beam current $I_{B}$; mass flow rate $Q$; emitter potential $\phi_{E}$; estimated potential $\phi_{J}$, velocity $v_{J}$ and diameter $D_{J}$ of the jet at the breakup point; and mean diameter of main droplets $\langle D\rangle$.

law, which supports this method for calculating the initial velocity and potential of droplets (Gamero-Castaño \& Hruby 2002). The lack of satellite droplets and the resulting reduced range of charge to mass ratios prevents the use of this method for the $46 \mathrm{nA}$ electrospray, and handicaps the estimate for the $58 \mathrm{nA}$ case. Figure 8 reveals that the voltage drop along the cone jet is roughly half of the available voltage while the terminal jet velocity is of the order of $100 \mathrm{~m} \mathrm{~s}^{-1}$. Table 1 gives currents and flow rates, emitter potentials and the estimated jet velocities and potentials.

The high sensitivity of the beam model to the initial velocity of droplets, a result to be derived in $\S 3.1$, underlines the need for information on the initial velocity distribution. We have shown that (3) can be used to estimate the mean initial velocity. In addition, the variance of the initial velocities can be estimated from the retarding potential distribution if the spread of the latter is not driven by Coulombic repulsion between droplets. If this were true, and due to the small spread of the charge to 
mass ratio (at least in the case of main droplets), the standard deviation of initial velocities would be proportional to the standard deviation of the retarding potential distribution:

$$
\sigma_{v_{0}} \cong \frac{\langle\xi\rangle}{\left\langle v_{0}\right\rangle} \sigma_{\phi_{R P}}=\frac{\langle\xi\rangle}{v_{J}} \sigma_{\phi_{R P}} .
$$

The negligible contribution of the electric interaction between droplets is a hypothesis that can be tested by solving the following 'line of point charges' model:

$$
\left.\begin{array}{c}
\ddot{\boldsymbol{x}}_{i}(t)=\frac{\xi_{i}}{4 \pi \varepsilon_{0}} \sum_{j=-K}^{K, j \neq i} q_{j} \frac{\boldsymbol{x}_{i}-\boldsymbol{x}_{j}}{\left(\boldsymbol{x}_{i}-\boldsymbol{x}_{j}\right)^{3}}, \\
\boldsymbol{x}_{i}(0)=\left\{\begin{array}{l}
\text { Random }[-1,1] R_{J} / \sqrt{2} \\
\operatorname{Random}[-1,1] R_{J} / \sqrt{2} \\
z
\end{array}\right\}, \dot{\boldsymbol{x}}_{i}(0)=0, \\
z=-\lambda_{o}+\lambda(i+1), \xi_{i}=\langle\xi\rangle, q_{i}=\langle q\rangle, i=-K, \ldots,-1, \\
z=0, \xi_{i}=\xi_{o}, q_{i}=q_{o}, i=0, \\
z=\lambda_{o}+\lambda(i-1), \xi_{i}=\langle\xi\rangle, q_{i}=\langle q\rangle, i=1, \ldots, K,
\end{array}\right\}
$$

The model consists of $2 K+1$ droplets placed along the $z$-axis, with random $y$ and $x$ initial offsets of the order of the jet radius $R_{J}$. A random sample of the number distribution of the electrospray droplets yields the central droplet, $i=0$; this droplet has a charge $q_{o}$ and a charge to mass ratio $\xi_{o}$. The diameter of the surrounding droplets, $i \neq 0$, is the mean diameter $\langle D\rangle$ of the main droplet distribution, while their charge and charge to mass ratio is that associated with $\langle D\rangle$; the droplets surrounding the central droplet in this line of point charges model are therefore the average main droplet of the electrospray. The initial $z$-separation between two average droplets is the critical wavelength $\lambda$ of the jet breakup, while the distance $\lambda_{o}$ between the central droplet and any of its two neighbouring droplets is the arithmetic mean of the central droplet wavelength and $\lambda$. We integrate in time this system of $2 K+1$ differential equations and stop when the potential energy of the group of droplets is $0.1 \%$ of the initial potential energy. At this point, the kinetic energy of the central droplet, divided by its charge, is its retarding potential induced by Coulombic interaction with neighbouring droplets. Figure 9 shows the retarding potential distributions of central droplets, constructed by solving the model 1500 times with central droplets sampled from the main droplet distribution and a value of 4 for $K$. A larger number of neighbouring droplets can be used, but this only increases the mean of the distribution and has little effect on its variance. Note that the standard deviations in figure 9 are one order of magnitude smaller than those measured in figure 5. Therefore neglecting the effect of interactions between droplets on the spread of the retarding potential distributions is a sensible approximation, and we will use (4) to estimate the standard deviation of their initial velocities. With the jet velocities computed in figure 8 , the standard deviations for the 69, 92 and $125 \mathrm{nA}$ electrospray beams are 5.8, 5.4 and $4.7 \mathrm{~m} \mathrm{~s}^{-1}$, respectively, i.e. $5.6 \%, 5.7 \%$ and $5.5 \%$ of the mean initial velocities. 


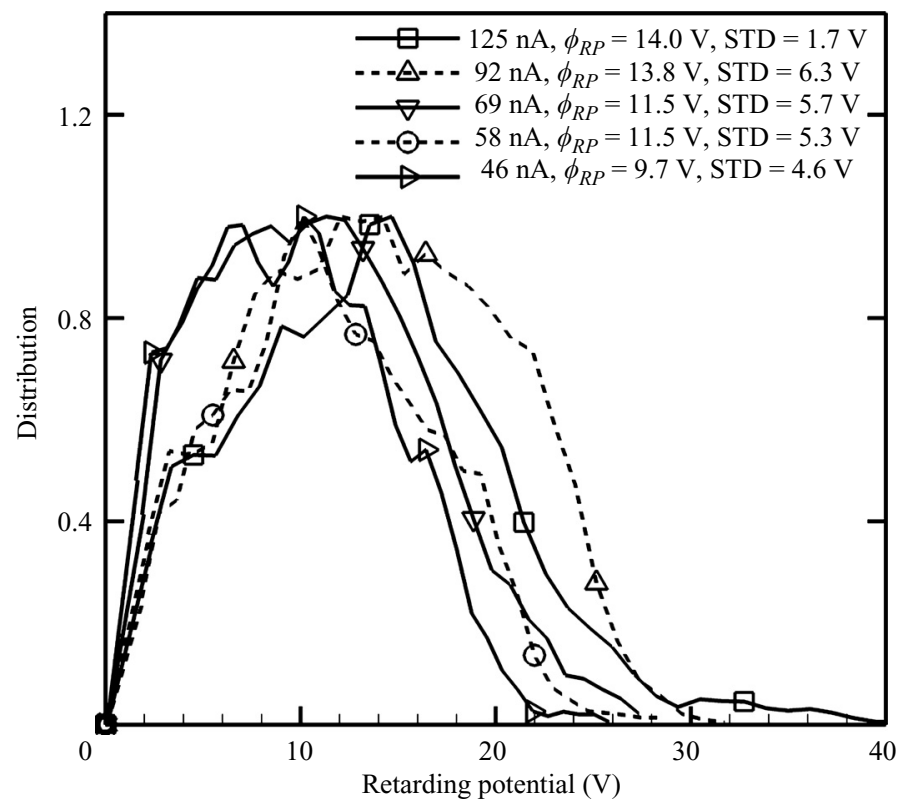

FIGURE 9. Estimated retarding potential distribution induced by Coulombic interaction between droplets. The standard deviations induced by this mechanism are much smaller than the standard deviations of the measured retarding potential distributions.

It is surprising that the experimental retarding potential distributions are so broad, especially in view of the negligible contribution from electrical interaction between droplets and the near constant charge to mass ratio of the main droplets. Our assumption of a fixed axial location for the breakup does not change this finding, because the jet is accelerated with minimal losses of mechanical energy, and therefore the retarding potential of the fluid along the jets is approximately constant regardless of where it breaks. It appears that the only reservoir of energy large enough to drive the broadness of the retarding potential distributions is the surface of the fluid, which changes considerably during the transition from jet to droplet. Proving whether the excess of surface energy is indeed the origin of the spread of retarding potentials and how it would be converted into kinetic energy is not essential for this paper. The relevant point is that Coulomb interaction between droplets cannot induce the spread of the retarding potential distributions, and therefore the latter can be used to estimate or at least bound the spread of the initial velocity distribution.

\section{Beam model}

The motion of an electrospray droplet is driven by the electric field induced by the external electrodes, together with the electric interaction with all other droplets in the beam. The latter makes the simultaneous integration of every droplet's equation of motion the natural method for reproducing the structure of the beam. However, this Lagrangian approach has several problems: first, the number of evaluations at each time step is proportional to the square of the number of droplets, bounding rather fast the number of trajectories that we can simulate; secondly, the improved accuracy resulting from the expensive tracking of individual droplets may not result in a more accurate solution because of uncertainties in parameters such as the initial velocity distribution; thirdly, it is incompatible with an analytical solution and we 


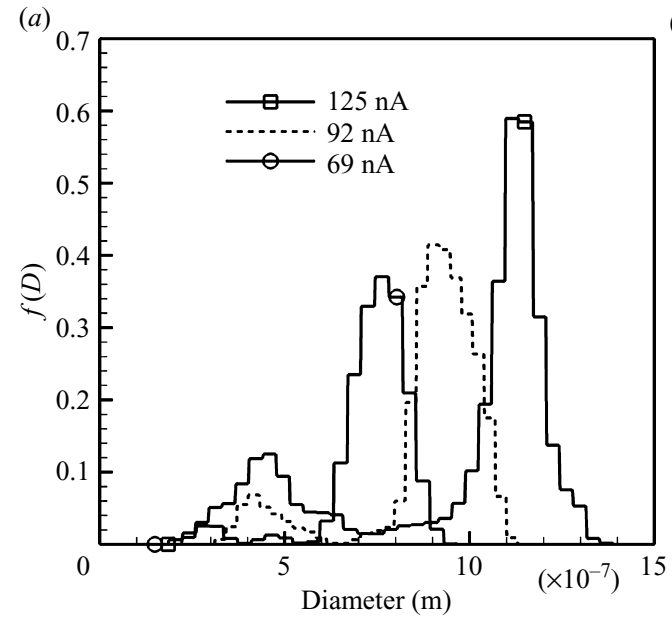

(b)

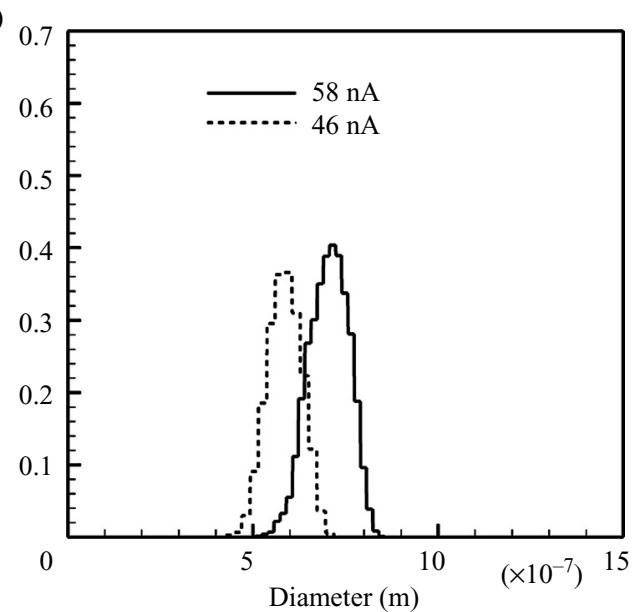

FIGURE 10. Experimental droplet diameter distributions. The largest beam currents display bi-modal distributions associated with main and satellite droplets. The beam current is the integral of $f(D)$.

are interested in the functional dependence between the structure of the electrospray and the properties of the droplets. This justifies the use of the time-independent Eulerian model described below, for which an approximate analytical solution can be found.

\subsection{Analytical model}

The ultimate goal of our model is to find the beam current density distribution $f\left(D, v_{0}, \theta\right)$ at the extractor plane, which is a function of the droplet diameter, its initial velocity $v_{0}$, and the inverse tangent $\theta$ of its trajectory at the extractor plane. $\theta$ is also referred to as the exit angle because, downstream of the extractor, the forces acting on a droplet are negligible, and its trajectory becomes a straight line. The beam current is, by definition, the multiple integral of $f\left(D, v_{0}, \theta\right)$ for all $D, v_{0}$ and $\theta$. We will assume that the charge and the charge to mass ratio of a droplet are functions of its diameter only, $q(D)$ and $\xi(D)$. The notation $f(x)$ and $f(x, y)$ stand for the definite integral of the original distribution throughout the ranges of two, and one, of its independent variables. For example, figure 10 shows the diameter distributions $f(D)$ of the 46, 58, 69, 92 and $125 \mathrm{nA}$ electrosprays, constructed with ICD droplet data and the beam current profiles. Using the experimental diameter distributions and a normal distribution for the droplet initial velocity (a choice justified in §2.4), the beam current distribution function is written as

$$
f\left(D, v_{0}, \theta\right)=\frac{1}{\sigma_{v} \sqrt{2 \pi}} \exp \left(-\left(\frac{v_{0}-v_{J}}{\sqrt{2} \sigma_{v}}\right)^{2}\right) f(D) h\left(\theta ; D, v_{0}\right),
$$

where $\sigma_{v}$ is the standard deviation of the initial velocity distribution. The angular density distribution $h\left(\theta ; D, v_{0}\right)$ is a parametric function of the droplet diameter and initial velocity. If $f\left(D, v_{0}, \theta\right)$ were known, $h\left(\theta ; D, v_{0}\right)$ could be constructed by varying $\theta$ in $f\left(D, v_{0}, \theta\right)$, while keeping $D$ and $v_{0}$ fixed. $h\left(\theta ; D, v_{0}\right)$ would be the resulting $\theta$-curve, normalized by its integral.

We will compute $h\left(\theta ; D, v_{0}\right)$ by solving the equations for the trajectory $R(z)$ (i.e. the radial component of the position vector in cylindrical coordinates) and axial velocity 
$v(z)$ of a droplet, as functions of the axial coordinate $z$ :

$$
\begin{gathered}
v^{2} \frac{\mathrm{d}^{2} R}{\mathrm{~d} z^{2}}+v \frac{\mathrm{d} v}{\mathrm{~d} z} \frac{\mathrm{d} R}{\mathrm{~d} z}=\xi\left(E_{r}^{s p c h}+E_{r}^{e x t}\right), \\
v \frac{\mathrm{d} v}{\mathrm{~d} z}=\xi E_{z}^{e x t}, \\
\boldsymbol{E}^{e x t}=-\nabla \phi^{e x t}, \\
R(0)=0, \quad R^{\prime}(0)=0, \quad v(0)=v_{0} .
\end{gathered}
$$

We separate the electric field into terms induced by the external electrodes $\left(\boldsymbol{E}^{\text {ext }}\right)$ and the space charge of the electrospray droplets $\left(\boldsymbol{E}^{\text {spch }}\right)$. This decomposition is an approximation motivated by the lack of information about the geometry of the cone-jet, and does not take into account the induced charge created in the electrodes and liquid bulk by the droplets. Furthermore, we assume that the variation of the trajectories with $z$ is sufficiently slow to make the axial component of $\boldsymbol{E}^{\text {spch }}$ negligible (Reiser 1994). The charge to mass ratio and the initial velocity of the droplet appear explicitly in the equations of motion. We will show that the droplet diameter has an important effect on $\boldsymbol{E}^{\text {spch }}$, and therefore the trajectory of a droplet will be a function of its diameter as well.

The structure of the beam could be computed using a continuous model of averaged beam parameters similar to the Navier-Stokes formulation in fluid mechanics. Poisson's equation for the averaged electric field would complement the equations for the averaged beam velocity and droplet distributions. Unlike our model, the continuum approach would have an exact expression for the averaged electric field, but is extremely complex: the model must account for the fundamental distributions of droplet diameter, charge and initial velocity in the sprays; the nonlinear inertial term must be retained; the nonlinear partial differential equations for the averaged velocity and droplet distributions are coupled to Poisson's equation, and the space charge distribution is not known a priori; besides the averaged electric field, modelling the Coulombic interaction between droplets also requires the addition of an ad hoc collision term; and probably, the characteristic length required to define averaged quantities in these low-density sprays will yield an insufficient spatial resolution. We are unaware of a mathematical formulation that includes all these complexities. If it is possible, it is apparent that the continuum formulation is not compatible with the analytical solution sought for our problem.

We estimate $\boldsymbol{E}^{\text {spch }}$ by using a line of point charges model similar to that described in §2.4. The droplet with a trajectory defined by (9)-(12) is the central droplet in the line of point charges model. The neighbouring droplets have the charge and diameter of the average main droplet, and form an infinite array that moves along the $z$-axis with the central droplet and keeps a zero radial coordinate. They induce an electric field on the central droplet at radial position $R$

$$
E_{r}^{\text {spch }}(R)=\frac{2\langle q\rangle}{4 \pi \varepsilon_{0}} \sum_{n=0}^{\infty} \frac{R}{\left[\left(\lambda_{o}+n \lambda\right)^{2}+R^{2}\right]^{3 / 2}} .
$$

The factor of 2 in the numerator accounts for the neighbouring droplets upstream of the central droplet. The diameters of the central and average droplets enter in this formula through $\lambda_{o}$ and $\lambda$, and therefore have an effect on the trajectory. We 


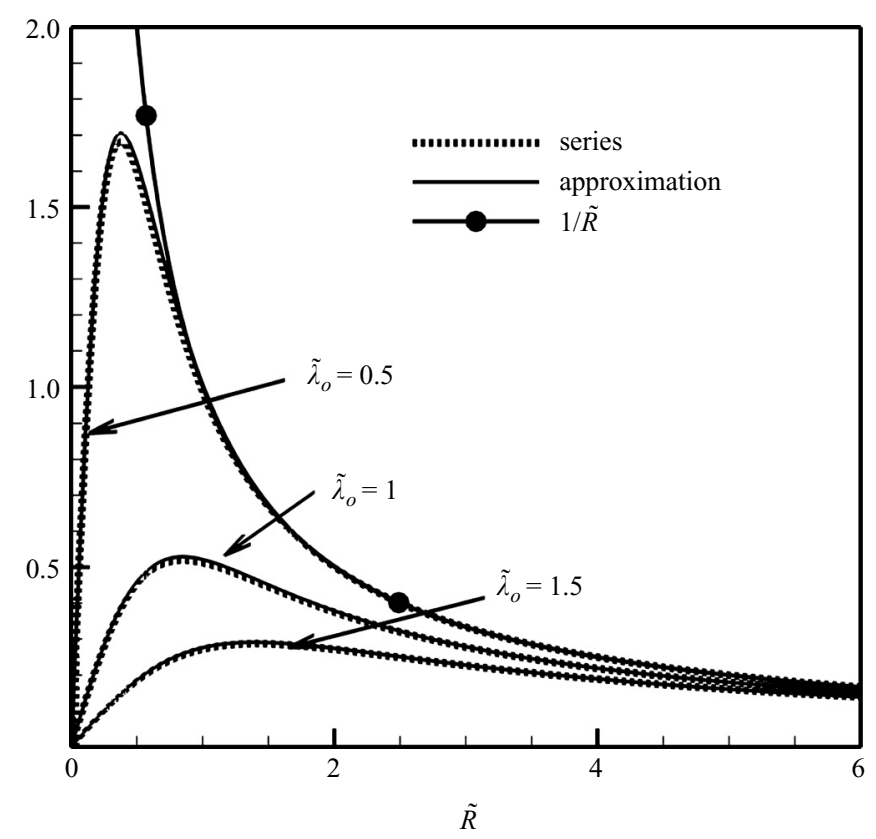

FIGURE 11. Electric field induced by a line of point charges: exact solution and approximations of the series $\sum_{n=0}^{\infty}\left(\tilde{R} /\left[\left(\tilde{\lambda}_{o}+n\right)^{2}+\tilde{R}^{2}\right]^{3 / 2}\right)$ for different droplet sizes, $\tilde{\lambda}_{o}$.

obtain an approximate value for the infinite series by separating the nearest droplet contribution, and integrating over $n$ for the remaining droplets

$$
\begin{aligned}
\sum_{n=0}^{\infty} \frac{\tilde{R}}{\left[\left(\tilde{\lambda}_{o}+n\right)^{2}+\tilde{R}^{2}\right]^{3 / 2}} & \cong \frac{\tilde{R}}{\left(\tilde{\lambda}_{o}^{2}+\tilde{R}^{2}\right)^{3 / 2}}+\int_{1 / 2}^{\infty} \frac{\tilde{R} \mathrm{~d} n}{\left[\left(\tilde{\lambda}_{o}+n\right)^{2}+\tilde{R}^{2}\right]^{3 / 2}} \\
& =\frac{\tilde{R}}{\left(\tilde{\lambda}_{o}^{2}+\tilde{R}^{2}\right)^{3 / 2}}+\frac{\sqrt{4 \tilde{R}^{2}+\left(1+2 \tilde{\lambda}_{o}\right)^{2}}-\left(1+2 \tilde{\lambda}_{o}\right)}{\tilde{R} \sqrt{4 \tilde{R}^{2}+\left(1+2 \tilde{\lambda}_{o}\right)^{2}}} .
\end{aligned}
$$

In this expression, $\lambda$ is used as the characteristic length to define the dimensionless $\tilde{\lambda}_{o}$ and $\tilde{R}$. Figure 11 plots the exact value of the series together with its approximation, for different values of $\tilde{\lambda}_{o}$. For satellite droplets, $\tilde{\lambda}_{o}$ is near the minimum possible value of 0.5 because of their small diameters relative to the average main droplet's. For main droplets, $\tilde{\lambda}_{o}$ distributes around 1 , typically between 0.7 and 1.4. Figure 11 shows that the approximation for the series is adequate for all physical values of $\tilde{\lambda}_{o}$. The electric field at the axis is zero and has a maximum near $\tilde{R}=1$. The smaller the droplet, the larger the maximum and the earlier it is reached. It is worth noting that $(a)$ a uniform distribution of charge along the axis generates a radial field proportional to $1 / \tilde{R}$; and $(b)$ the line of point charges model asymptotes relatively fast to this $1 / \tilde{R}$ limit, especially for the smallest droplets. The convergence fails for small $\tilde{R}$ because $1 / \tilde{R}$ is singular at $\tilde{R}=0$.

The right-hand side of (14) multiplied by $\kappa I_{B} \lambda / v_{J}$ instead of $\langle q\rangle$ is our expression for the space charge field:

$$
E_{r}^{s p c h}(\tilde{R})=\frac{\kappa I_{B}}{2 \pi \varepsilon_{0} v_{J} \lambda}\left[\frac{\tilde{R}}{\left(\tilde{\lambda}_{o}^{2}+\tilde{R}^{2}\right)^{3 / 2}}+\frac{\sqrt{4 \tilde{R}^{2}+\left(1+2 \tilde{\lambda}_{o}\right)^{2}}-\left(1+2 \tilde{\lambda}_{o}\right)}{\tilde{R} \sqrt{4 \tilde{R}^{2}+\left(1+2 \tilde{\lambda}_{o}\right)^{2}}}\right] .
$$


The charge of the average main droplet $\langle q\rangle$ is approximately equal to $I_{B} \lambda / v_{J}$ from conservation of charge in the jet. The new term $\kappa$, referred to as strength factor, is an input parameter with a value between 0 and 1 and remains constant along the droplet trajectory. The use of the strength factor is motivated by the fact that in a real breakup, the space charge forces felt by identical droplets will vary within an interval ranging from zero to a maximum value of the order of the estimate given by the line of point charges model. This range of forces causes the angular distribution of identical droplets observed in experiments.

Upon inserting the space charge field, the dimensionless equations of motion are

$$
\begin{aligned}
& \tilde{v}^{2} \frac{\mathrm{d}^{2} \tilde{R}}{\mathrm{~d} \tilde{z}^{2}}+\tilde{v} \frac{\mathrm{d} \tilde{v}}{\mathrm{~d} \tilde{z}} \frac{\mathrm{d} \tilde{R}}{\mathrm{~d} \tilde{z}}=\beta\left[\frac{\tilde{R}}{\left(\tilde{\lambda}_{o}^{2}+\tilde{R}^{2}\right)^{3 / 2}}+\frac{\sqrt{4 \tilde{R}^{2}+\left(1+2 \tilde{\lambda}_{o}\right)^{2}}-\left(1+2 \tilde{\lambda}_{o}\right)}{\tilde{R} \sqrt{4 \tilde{R}^{2}+\left(1+2 \tilde{\lambda}_{o}\right)^{2}}}\right]+\delta \tilde{E}_{r}^{e x t} \\
& \tilde{v} \frac{\mathrm{d} \tilde{v}}{\mathrm{~d} \tilde{z}}=\delta \tilde{E}_{z}^{e x t} .
\end{aligned}
$$

We use the jet velocity, the mean breakup wavelength $\lambda$, and the axial component of the external electric field at the jet breakup $E_{J}$ to make the variables dimensionless. Near the emission point $\tilde{E}_{z}^{\text {ext }}$ is $O(1)$. Furthermore, $\tilde{E}_{r}^{\text {ext }}$ near the axis is negligible because the emission point is far from the singular vertex of the Taylor cone (the voltage drops along the cone-jet in table 1 are significant) and the electrospray source is axisymmetric. $E_{J}$ can be estimated by solving the Laplace equation in prolate spheroidal coordinates (Morse \& Feshbach 1953):

$$
\begin{gathered}
\phi(\zeta, \eta, \vartheta)=\phi_{J} \frac{\operatorname{arctanh}(\eta)}{\operatorname{arctanh}\left(\eta_{E}\right)}, \\
E_{J}=\frac{2 E_{\eta}\left(1, \eta_{E}, \vartheta\right)}{a}=\phi_{J} \frac{2}{a \operatorname{arctanh}\left(\eta_{E}\right)} \frac{1}{1-\eta_{E}^{2}} .
\end{gathered}
$$

The surface $\zeta=$ onstant is a prolate spheroid with interfocal distance $2 a$, major axis $\zeta a$, and minor axis $a\left(\zeta^{2}-1\right)^{1 / 2}$; the surfaces $\eta=$ constant are two sheets of a hyperboloid of revolution with foci at $z= \pm a$, asymptotic to the cone which has its generating line at an angle $\vartheta=\cos ^{-1} \eta$ to the $z$-axis, and the surface $\vartheta=\mathrm{constant}$ is a plane through the $z$-axis at an angle $\vartheta$ to the $(x, z)$-plane. In this solution by Martínez-Sánchez (2004) the surfaces of both the capillary emitter and the extractor are regarded as hyperboloids, i.e. surfaces of constant $\eta$. The $\eta$-coordinate of the extractor plane is zero. The foci of the emitter hyperboloid are at $z= \pm a$, where $a$ is the distance between the emitter and the extractor plane. We make the radius $R_{E}$ of the cylindrical needle equal to the radius of curvature $\left(a-a \eta_{E}^{2}\right) / 2 \eta_{E}$ of the emitter hyperboloid at the axis, to obtain the value of $\eta$ at the emission point, $\eta_{E}=0.983$ ( $a=3.17 \mathrm{~mm}, R_{E}=0.11 \mathrm{~mm}$ for our geometry). The potential field can be visualized by noticing that the equipotential surfaces between the emitter and extractor are also hyperboloids with identical foci. As the value of the hyperboloid potential decreases, its intersection with the axis approaches the extractor plane, and its radius of curvature at the axis increases. With these values for $\eta_{E}$ and $a$, our estimate of $E_{J}$ is equal to the factor $3895 \mathrm{~m}^{-1}$ multiplied by the electric potential at the breakup.

The parameters $\beta$ and $\delta$ are the ratios between the work done on a droplet by electrostatic forces (space charge in the case of $\beta$, external field in the case of $\delta$ ) along a mean breakup wavelength $\lambda$, divided by the characteristic kinetic energy of 
the droplet

$$
\beta=\frac{\xi \kappa I_{B}}{2 \pi \varepsilon_{0} v_{J}^{3}}, \quad \delta=\frac{\xi \lambda E_{J}}{v_{J}^{2}} .
$$

Both $\beta$ and $\delta$ are small numbers. $\delta$ must be small because the fluid in the jet gains kinetic energy forced by an electric field larger than $E_{J}$, acting along a distance many times greater than $\lambda$. For $\kappa=1, \beta$ is bigger than $\delta$ because the radial component of the electric field at the jet perimeter, induced by its surface charge, is larger than the axial component of the electric field. Typical values of $\delta$ and $\beta$ for the $92 \mathrm{nA}$ beam are 0.0071 and 0.011 , respectively $\left(\kappa=1, \xi=I_{B} / Q\right)$.

The scales of $\beta, \delta, \tilde{E}_{z}^{\text {ext }}$ and $\tilde{E}_{r}^{\text {ext }}$ lead to these simplified equations valid near the emission point

$$
\begin{gathered}
\tilde{v}_{N}^{2} \frac{\mathrm{d}^{2} \tilde{R}_{N}}{\mathrm{~d} \tilde{z}^{2}}=\beta\left[\frac{\tilde{R}_{N}}{\left(\tilde{\lambda}_{o}^{2}+\tilde{R}_{N}^{2}\right)^{3 / 2}}+\frac{\sqrt{4 \tilde{R}_{N}^{2}+\left(1+2 \tilde{\lambda}_{o}\right)^{2}}-\left(1+2 \tilde{\lambda}_{o}\right)}{\tilde{R}_{N} \sqrt{4 \tilde{R}_{N}^{2}+\left(1+2 \tilde{\lambda}_{o}\right)^{2}}}\right], \\
\tilde{v}_{N}(\tilde{z})=\frac{v(0)}{v_{J}}=\tilde{v}_{N} .
\end{gathered}
$$

These equations describe the initial expansion of the beam forced by space charge repulsion. The importance of this initial space-charge-dominated region originates from its substantial independence from the external field: the trajectory of a droplet in the space charge region is an intrinsic property of the electrospray determined by parameters of the jet breakup. To calculate a global analytical solution valid between the emission point and the extractor plane, we would require an external region where (16)-(17) could be simplified, obtain approximate analytical solutions for both regions, and asymptotically match them along an intermediate region of common validity. Unfortunately, this strategy is not possible for two reasons: first, there is not an external region where (16)-(17) can be significantly simplified, i.e. most terms must be retained; and secondly, the external electric field is intrinsically two-dimensional, making (16)-(17) nonlinear. In view of the unfeasibility of a global analytical solution, we will look for a solution based on the initial region equations, combined with a criterion for estimating and defining a 'boundary' for the initial region. We will show that for the typical emitter-extractor geometry shown in figure 2 and the relatively near-axis trajectories of these sprays, the tangent of the trajectory does not change much downstream of the initial region boundary. Thus the solution of (21), evaluated at the boundary of the initial region, provides a good estimate of the exit angle of the droplet.

Equation (21) does not have a solution for $\tilde{R}_{N}(z)$ in terms of elementary functions. However, a first integral yields the tangent of the droplet trajectory, $\tan \left(\theta_{N}\right)=\mathrm{d} \tilde{R}_{N} / \mathrm{d} \tilde{z}$ :

$$
\tan \left(\theta_{N}\right)=\sqrt{\frac{2 \beta}{\tilde{v}_{N}^{2}}} \sqrt{\ln \left(\frac{\sqrt{4 \tilde{R}_{N}^{2}+\left(1+2 \tilde{\lambda}_{o}\right)^{2}}+1+2 \tilde{\lambda}_{o}}{2+4 \tilde{\lambda}_{o}}\right)+\frac{1}{\tilde{\lambda}_{o}}-\frac{1}{\sqrt{\tilde{R}_{N}^{2}+\tilde{\lambda}_{o}^{2}}} .}
$$

The validity of this solution breaks down when the weakening space charge force cannot prevent the inward bending of the droplet trajectory caused by its axial acceleration, i.e. when the $\tilde{v}(\mathrm{~d} \tilde{v} / \mathrm{d} \tilde{z})(\mathrm{d} \tilde{R} / \mathrm{d} \tilde{z})$ term of (16) becomes comparable to the right-hand side of (21). We impose this condition to define the boundary $\tilde{R}_{N}^{*}$ of the 
region dominated by space charge. $\tilde{R}_{N}^{*}$ is implicitly given by the algebraic equation

$$
\begin{aligned}
& \ln \left(\frac{\sqrt{4 \tilde{R}_{N}^{* 2}+\left(1+2 \tilde{\lambda}_{o}\right)^{2}}+1+2 \tilde{\lambda}_{o}}{2+4 \tilde{\lambda}_{o}}\right)+\frac{1}{\tilde{\lambda}_{o}}-\frac{1}{\sqrt{\tilde{R}_{N}^{* 2}+\tilde{\lambda}_{o}^{2}}} \\
& =\frac{\beta \tilde{v}_{N}^{2}}{2 \delta^{2}}\left[\frac{\tilde{R}_{N}^{*}}{\left(\tilde{\lambda}_{o}^{2}+\tilde{R}_{N}^{* 2}\right)^{3 / 2}}+\frac{\sqrt{4 \tilde{R}_{N}^{* 2}+\left(1+2 \tilde{\lambda}_{o}\right)^{2}}-\left(1+2 \tilde{\lambda}_{o}\right)}{\tilde{R}_{N}^{*} \sqrt{4 \tilde{R}_{N}^{* 2}+\left(1+2 \tilde{\lambda}_{o}\right)^{2}}}\right]^{2} .
\end{aligned}
$$

The value of $\tilde{R}_{N}^{*}$ inserted into (23) yields the tangent of the droplet trajectory at the boundary of the space charge region, $\theta_{N}^{*}$.

Before proceeding with the description of model results, let us summarize the variables and parameters used by the model. The dependent variables in the trajectory (9)-(12) are the radial position $R(z)$ and axial velocity $v(z)$ of the droplet. The independent variable is the axial position $z$. The electric field $\boldsymbol{E}^{\text {ext }}(\boldsymbol{x})$ induced by the external electrodes is a dependent variable in these equations, but only the axial component $E_{J}$ at the jet breakup is required for the simplified equations near the emission point. The solution is also a function of the following parameters of the droplet for which the trajectory is computed: charge to mass ratio $\xi$, initial axial velocity $v_{0}$, and breakup wavelength, which upon combination with $\lambda$ yields $\lambda_{o}$. Additional input parameters are the beam current $I_{B}$, the jet velocity $v_{J}$ and potential $\phi_{J}$ at the breakup, and the average breakup wavelength $\lambda$. The values of these parameters are either measured or inferred from experimental measurements of the electrosprays. We use these parameters to non-dimensionalize the variables of the model and to define the dimensionless numbers $\beta$ and $\delta$. The model also includes the definition of the strength factor $\kappa$. Although $\kappa$ ranges between 0 and 1 , neither the analytical model nor measurements can provide its value. At this stage, the strength factor must be regarded as a free parameter which must be specified a priori before computing the trajectory associated with it. Section 3.2 argues how to estimate $\kappa$ for the analytical model, while $\S 3.3$ provides a physical law for computing $\kappa$ when the trajectories of many droplets are integrated simultaneously.

\subsection{Analytical model results and comparison with experimental observations}

Figure 12 shows a comparison between $\theta_{N}$ and the 'exact' solution obtained by integrating numerically (16)-(17). We consider two droplets of the $92 \mathrm{nA}$ electrospray, the average main droplet $\left(\tilde{\lambda}_{o}=1, \beta=0.0097, \delta=0.0061\right)$ and a typical satellite droplet $\left(\tilde{\lambda}_{o}=0.56, \beta=0.032, \delta=0.020\right)$. The droplets are accelerated by the full force of the line of point charges model $(\kappa=1)$ and by the external electric field solved in prolate spheroidal coordinates. A small but non-zero initial value for the droplet radial position, $\tilde{R}(0)=0.01$, is imposed to allow the separation of its trajectory from the axis (the sensitivity of the trajectory to this parameter is small, owing to the radial dependence of the space charge force). The trajectory is integrated up to the extractor plane $(\tilde{z}=1011)$ to compute the full effect of the external electric field. Figure 12 also shows the 'exact' trajectory $\tilde{z}(\tilde{R})$ and the value of the exit angle $\theta_{N}^{*}$. The largest increase in the inverse tangent happens in a short initial area within a radius of approximately ten wavelengths $\lambda$. The exact solution and $\theta_{N}$ compare well in this interval, although $\theta_{N}$ is always larger because of the neglected axial acceleration. Beyond the boundary of the space charge region, defined by the pair $\left\{\tilde{R}_{N}^{*}, \theta_{N}^{*}\right\}$, the inverse tangent may slightly increase or decrease depending on the angle formed by the velocity of the 

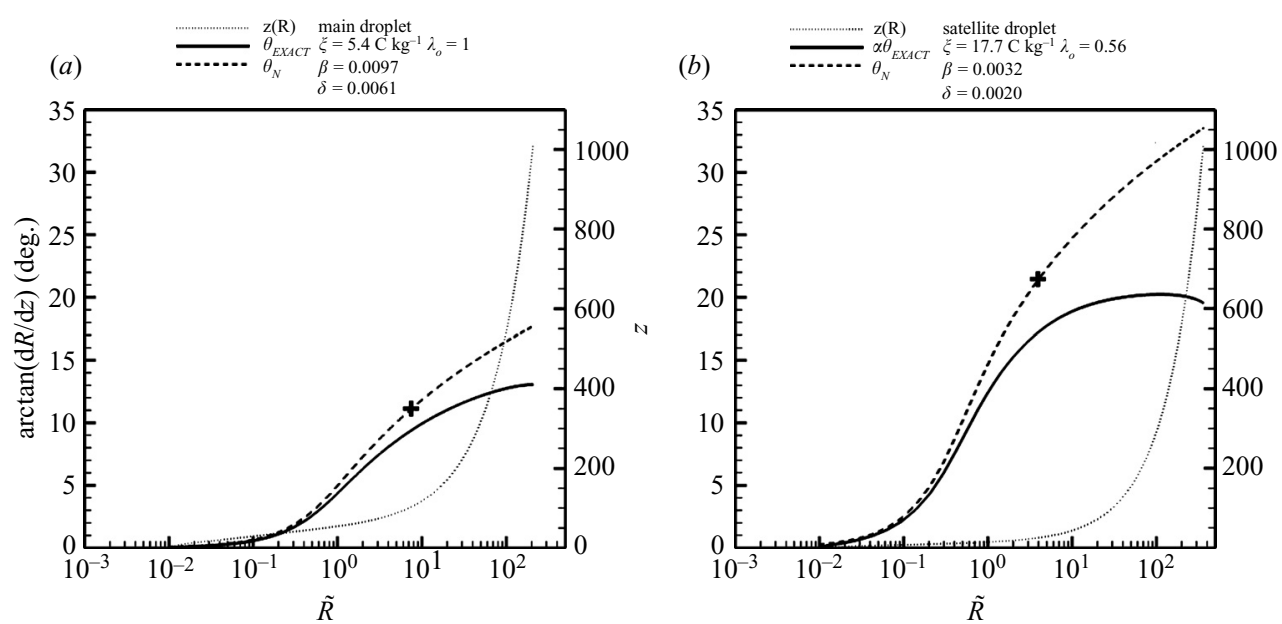

Figure 12. Numerical solution $\left(z(R)\right.$ and $\left.\theta_{E X A C T}\right)$ of (16) and (17) and approximate solution $\left(\theta_{N}\right)$ for the inverse tangent of the trajectory, (23). The pair $\left\{\tilde{R}_{N}^{*}, \theta_{N}^{*}\right\}$, identified by the symbol + , defines the boundary for the space-charge-dominated region and can be used as an estimate of the exit angle of the trajectory at the extractor plane. $I_{B}=92 \mathrm{nA}, \kappa=1$.

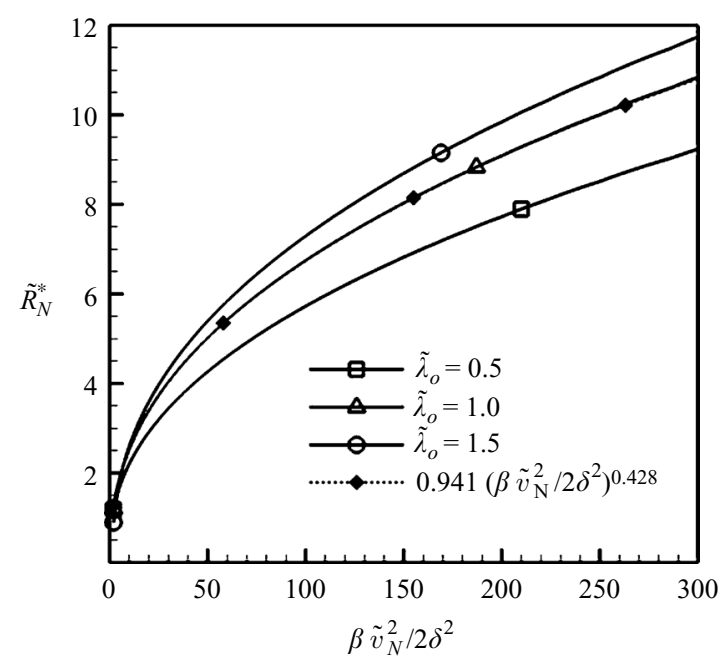

FIGURE 13. Radial boundary of the space-charge-dominated region defined by (24).

droplet and the external electric field. However, despite this contribution from the external field, $\theta_{N}^{*}$ is a good estimate of the exit angle at the extractor plane.

Figure 13 shows $\tilde{R}_{N}^{*}$ as a function of both $\tilde{\lambda}_{o}$ (i.e. droplet size effect), and the group $\beta \tilde{v}_{N}^{2} / 2 \delta^{2}$. For example, $\beta \tilde{v}_{N}^{2} / 2 \delta^{2}$ varies from $0(\kappa=0)$ to $139(\kappa=1)$ for the main droplets of the $92 \mathrm{nA}$ electrospray, while the upper bound for its satellite droplets is approximately 25 . $\tilde{R}_{N}^{*}$ increases with both $\beta \tilde{v}_{N}^{2} / 2 \delta^{2}$ and $\tilde{\lambda}_{o}$. Its range is mostly determined by the span of $\beta \tilde{v}_{N}^{2} / 2 \delta^{2}$, while the contribution of $\tilde{\lambda}_{o}$ is less significant. Figure 13 also displays a simple and accurate function for $\tilde{R}_{N}^{*}$ and $\tilde{\lambda}_{o}=1$, $\tilde{R}_{N}^{*}=0.941\left(\beta \tilde{v}_{N}^{2} / 2 \delta^{2}\right)^{0.428}$. 


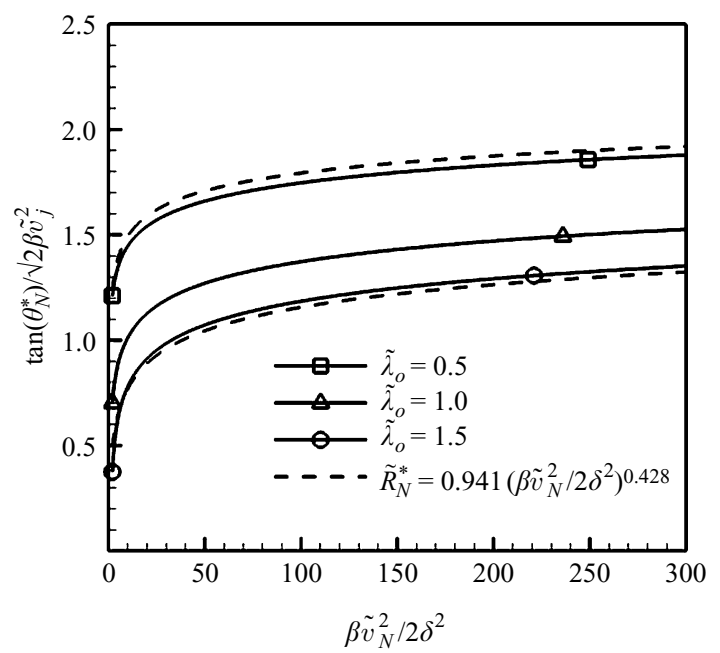

FigurE 14. Droplet size factor in (23) for the exit angle.

The expression for the tangent of $\theta_{N}^{*}$ can be separated into two components: a 'controlling factor' $\sqrt{2 \beta / \tilde{v}_{N}^{2}}$, and a 'droplet size factor' (the remaining terms on the right-hand side of $(23))$. The controlling factor varies between $0(\kappa=0)$ and 0.14 $(\kappa=1)$ for the main droplets of the $92 \mathrm{nA}$ electrospray, whereas its upper limit for a typical satellite droplet is 0.33 . Figure 14 plots the droplet size factor as a function of $\beta \tilde{v}_{N}^{2} / 2 \delta^{2}$ and $\tilde{\lambda}_{o}$. With the exception of droplets with very small polar angles (i.e. with small values of $\beta \tilde{v}_{N}^{2} / 2 \delta^{2}$ ), the droplet size factor is near or slightly larger than one. In contrast to $\tilde{R}_{N}^{*}$, the droplet size factor depends mostly on $\tilde{\lambda}_{o}$ and introduces a diameter dependency on the droplet exit angle. Thus, a satellite droplet will have a larger exit angle than a main droplet, not only because of the charge to mass ratio effect in the controlling factor $\sqrt{2 \beta / \tilde{v}_{N}^{2}}$, but also because its much smaller diameter increases the droplet size factor. Physically, this is due to the close proximity of the nearest droplet at the breakup (the main droplet from which the satellite detaches), and the resulting enhancement of the repulsive electric field acting on the satellite droplet. The droplet size factor also contributes to the weak angular separation of main droplets by diameters, especially for the electrosprays with narrowest charge to mass ratio distributions. Finally, the actual dependence of $\tilde{R}_{N}^{*}$ on $\tilde{\lambda}_{o}$ can be neglected when computing $\theta_{N}^{*}$. This is illustrated in figure 14 by the dashed curves calculated with the fitting of $\tilde{R}_{N}^{*}$ for $\tilde{\lambda}_{o}=1$, which fall near the exact solutions.

Equations (23) and (24) for $\kappa=1$ provide an estimate of the maximum exit angle. As $\kappa$ is varied from 1 to 0 , the same droplet will exit the space charge region with a lower trajectory. However, these equations do not determine the fraction of droplets that are associated with an exit angle, i.e. they do not yield the angular distribution $h\left(\theta ; D, v_{0}\right)$. We will compute $h\left(\theta ; D, v_{0}\right)$ by guessing a relationship between $\kappa$ and the integral of $h$ on $\theta, H\left(\theta ; D, v_{0}\right)$. Because the exit angle of a droplet with diameter $D$ and initial velocity $v_{0}$ is a function of the free parameter $\kappa$, a change of variables leads to $H\left(\kappa ; D, v_{0}\right)$. In this context, the cumulative distribution function $H$ is the fraction of beam current whose droplet trajectories are associated with a fraction of the space charge field equal to, or smaller than $\kappa$. Both $H$ and $\kappa$ vary between 0 and 1 . Although the problem of interaction between individual droplets must be solved to compute 
$H\left(\kappa ; D, v_{0}\right)$, the experimental profiles in figure 3 suggest that $H$ is approximately equal to $\kappa, H \cong \kappa$, at least for the main droplets. To show this, let us consider the beam profile $I_{\Omega}$ (current per unit solid angle) for the $46 \mathrm{nA}$ beam, and assume that all droplets are identical (based on the narrow diameter and $\xi$ distributions). When $H=\kappa$, (23) shows that $I_{B} H$ is approximately proportional to $\theta_{N}^{2}$ :

$$
I_{B} H=\frac{\pi \varepsilon_{0} v_{J}^{3} \tilde{v}_{N}^{2} / \xi}{\ln \left(\frac{\sqrt{4 \tilde{R}_{N}^{2}+9}+3}{6}\right)+1-\frac{1}{\sqrt{\tilde{R}_{N}^{2}+1}}} \tan ^{2}\left(\theta_{N}\right) \cong K \theta_{N}^{2} .
$$

This expression is a rearrangement of the terms in (23) particularized for a beam of identical droplets, $\tilde{\lambda}_{o}=1$. We use the small-angle approximation for the tangent function. $I_{B} H$ is the current flowing within the polar angle $\theta_{N}$, a quantity that can be obtained from the experimental profile $I_{\Omega}$ :

$$
I_{B} H(\theta)=\int_{0}^{\theta_{N}} 2 \pi I_{\Omega} \sin (x) \mathrm{d} x \cong \int_{0}^{\theta_{N}} 2 \pi I_{\Omega} x \mathrm{~d} x .
$$

Since the experimental profile resembles a rectangle function, $I_{B} H(\theta)$ is approximately a parabola just as (25) predicts. Thus, it is reasonable to assume that $H \cong \kappa$ for the main droplets which are similar. On the contrary, $H(\kappa)$ should behave differently for the smaller satellite droplets, with $H^{\prime}(\kappa)$ peaking at a value of the strength factor near 1. This stems from the larger charge to mass ratio and smaller diameter of the satellite droplets, together with the radial shape of the electrical potential induced by the space charge (it is maximum at the beam axis): droplets at the axis are in a position of unstable equilibrium, and any disturbance knocks them off radially. Satellite droplets move away from the axis faster than main droplets because of the larger $\xi$, compounded by the closer proximity of its two neighbouring droplets. Thus, almost any satellite droplet will rapidly be in a radial position above those of main droplets and, because the latter carry most of the beam current, they will be driven by a large fraction of the maximum space charge field. With this caveat, and to advance further, we will consider the simple case $H=\kappa$ for every droplet, and compute $h(\theta$; $D, v_{0}$ ) with (23) and (24), the substitution of $\kappa$ for $H$, and the relation between cumulative and density distributions $h\left(\theta ; D, v_{0}\right)=\partial_{\theta} H\left(\theta ; D, v_{0}\right)$.

Figure 15 shows the current angular distributions $f(\theta)$ for the 125, 92 and $69 \mathrm{nA}$ beams, computed with both the model and experimental profiles. The distributions for the two smallest beam currents are not plotted because of the uncertainties in the velocity and the potential at the jet breakup. We have found that the prolate spheroidal coordinates solution for the external electric field overestimates $E_{J}$ by a factor greater than two: when solving the Laplace equation numerically for the emitter geometry free of space charge, the axial electric fields at the jet breakup point (which is identified by the value of its potential) are $1.79 \times 10^{6}, 1.25 \times 10^{6}$ and $1.70 \times 10^{6} \mathrm{~V} \mathrm{~m}^{-1}$ for the 125,92 and $69 \mathrm{nA}$ beams, while the prolate spheroidal coordinates estimates are $3.98 \times 10^{6}, 3.39 \times 10^{6}$, and $3.78 \times 10^{6} \mathrm{~V} \mathrm{~m}^{-1}$. Figure 15 displays the angular distributions associated with both sets of electric fields to illustrate the effect of $E_{J}$. The shapes of the experimental and computed distributions agree reasonably well, especially for the more accurate value of $E_{J}$. A difference in $E_{J}$ by a factor greater than 2 translates into a $20 \%$ shift of the main droplet beam edge. $E_{J}$ influences the solution through its presence in the denominator of the dimensionless number $\delta$. Thus, the external electric field is a factor in the definition of the boundary 
(a)

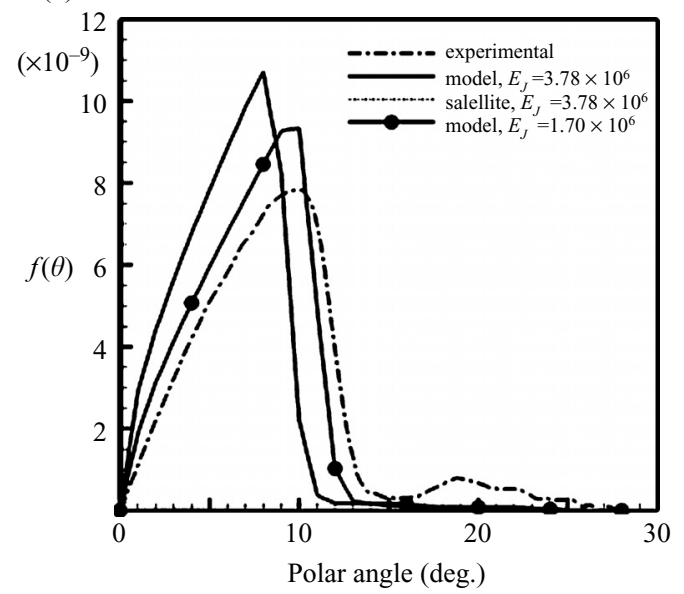

(b)

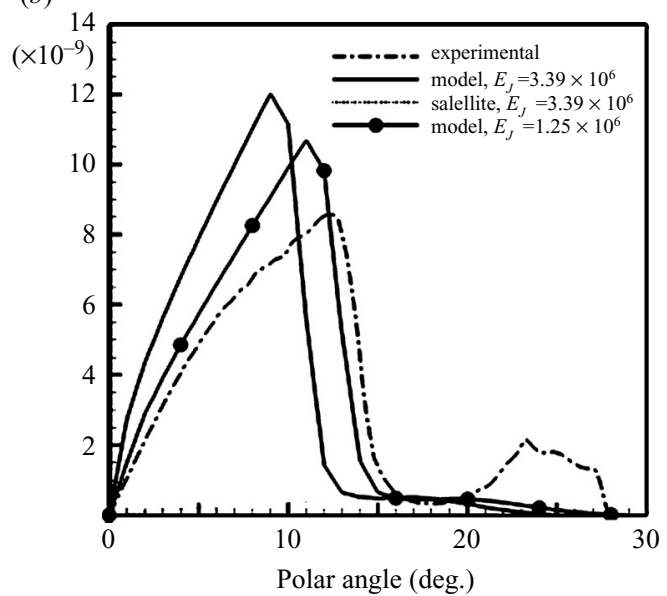

(c)

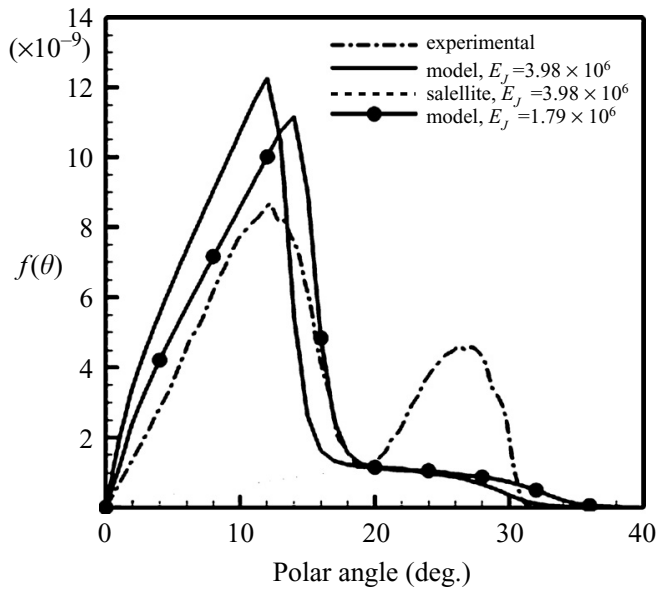

FIGURE 15. Experimental angular profiles and analytical solutions in the space charge region, for the three higher beam currents. (a) $I_{B}=69 \mathrm{nA}$; (b) $92 \mathrm{nA}$; (c) $125 \mathrm{nA}$. The analytical solutions are computed for two values of the axial component of the electric field.

of the space charge region and as such has an effect on the exit angle, but it only enters through the nearly constant droplet size factor on the right-hand side of (23). An important divergence in figure 15 is the presence of satellite droplets at small polar angles in the model distribution, which is not observed in the experimental data: the actual sprays have a sharp angular separation of main and satellite droplets. This discrepancy is due to the use of $H=\kappa$ for satellite droplets, a shortcoming of the analytical solution already explained.

We use the prolate spheroidal estimate of $E_{J}$ in the remaining graphs of $\S 3.2$. We will use a more accurate external electric field in $\S 3.3$, where a full numerical solution is computed. For now, we are interested in the trends exposed by the analytical solution, and for which the less accurate but simpler form of $E_{J}$ suffices. Figure 16 shows measured and model $f(D, \theta)$ distributions for the $125 \mathrm{nA}$ beam. They are plotted as a function of the droplet diameter, for different values of the exit polar angle. We have divided $f(D, \theta)$ by $f(\theta)$ to normalize the heights of the curves. We consider only main droplets in the model distributions to eliminate the distortion 

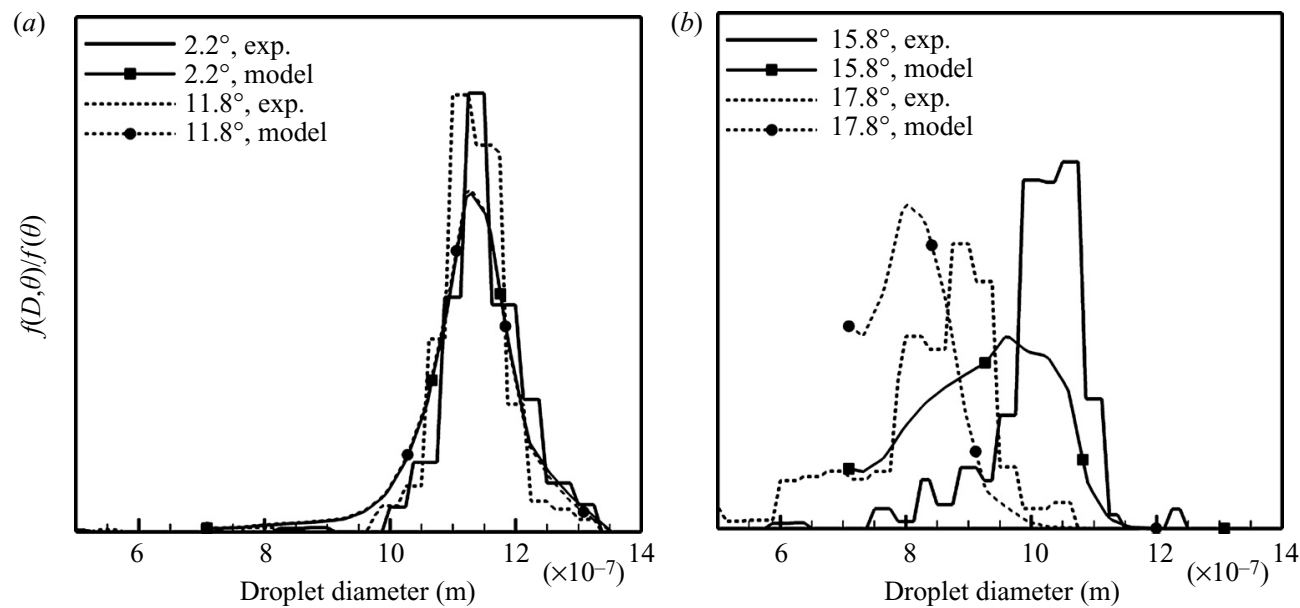

FIGURE 16. Experimental diameter distributions and analytical solutions at different polar angles $\left(I_{B}=125 \mathrm{nA}\right)$. The angular separation of main droplets by diameters is important along the inner beam edge.

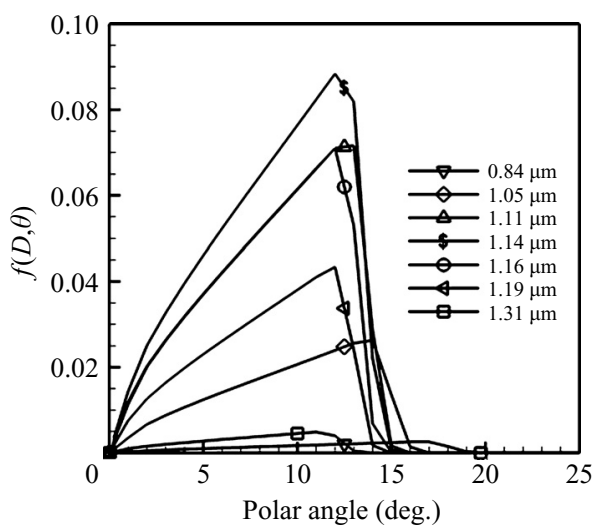

FIGURE 17. Analytical angular distributions for different main droplet diameters $\left(I_{B}=125 \mathrm{nA}\right)$. The initial lack of angular separation by droplet diameters is a result of the initial quasi-linearity of the distributions.

caused by the unphysical presence of satellite droplets at small polar angles. A remarkable feature of this figure is the near constancy of the diameter distributions throughout most of the angular range of the main droplets, a characteristic already hinted by figure 7. For example, the experimental distributions at $2.2^{\circ}$ and $11.8^{\circ}$ are nearly identical, while the computed distributions are indistinguishable. Beyond approximately $14^{\circ}$, and coinciding with the edge of the main droplet beam in figure 3 , the mean of the diameter distributions shifts to smaller diameters. This phenomenon is better explained by the shape of the $f(D, \theta)$ distributions when they are plotted as a function of $\theta$, as shown in figure 17. For a given diameter, $f(D, \theta)$ starts at the origin of coordinates, it is approximately a straight line throughout most of its angular range, and transitions into a sharply falling edge once a critical angle is reached. The finite slope of the edge is a consequence of the integration of $f\left(D, v_{0}, \theta\right)$ in velocity space (the trailing edge of $f\left(D, v_{0}, \theta\right)$ at fixed diameter and initial velocity is a vertical line). The critical angle of a droplet and therefore its angular range increases with decreasing 


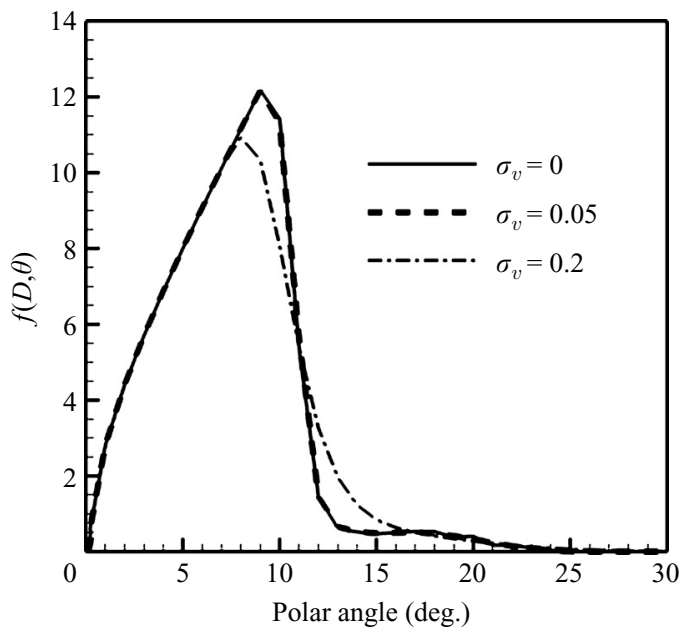

FIGURE 18. Computed angular profiles for different values of the initial velocity standard deviation $\left(I_{B}=92 \mathrm{nA}\right)$.

diameter. According to figure 17, the fraction of droplets with a given diameter will remain constant at increasing polar angle because its angular distribution is a straight line. As a result, the shapes of the diameter distributions will be identical regardless of $\theta$. This constancy of the diameter distributions ceases beyond the critical angle of the largest main droplet, which is approximately $13.8^{\circ}$ for the $125 \mathrm{nA}$ beam. From this point on, there is a separation of main droplets by diameters because the larger droplets disappear gradually from the spray, reducing the mean diameter of the distributions as the polar angle increases. Note that the angular insensitiveness predicated by the model is a consequence of the square root in the controlling factor of (23), together with the assumed proportionality between cumulative current and strength factor for main droplets, $H=\kappa$. Because this model prediction agrees with the experimental observation, we conclude that the $H=\kappa$ simplification for main droplets is indeed a good approximation (the original argument justifying this equality was based on the rectangular shape of the profile of a beam made of identical droplets).

In the previous graphs, we have used a standard deviation $\sigma_{v}=0.05 v_{J}$ for the initial velocity distributions. The $5 \%$ value is based on the analysis of the retarding potential curves described in $\S 2.4$. Figure 18 shows angular distributions for different standard deviations. This parameter has a minor effect on the shape of the angular distribution at the values typical of these electrosprays. Broader initial velocity distributions (standard deviations of the order of $20 \%$ of the jet velocity) are required to smooth the beam profiles significantly, in particular, to smooth the sharp edge of the main droplet beam. The dimensionless number $\beta$ in the controlling factor of the exit angle is inversely proportional to the cube of the characteristic initial velocity $v_{J}$, while the droplet size factor has a weaker dependence on $v_{J}$. Therefore, the angular distribution will significantly shift to larger angles for decreasing values of the jet breakup velocity. The spread of the initial velocities, reflected on the parameter $\tilde{v}_{N}$, is also part of the controlling and droplet size factors. For narrow velocity distributions, $\tilde{v}_{N}$ does not depart much from 1, and the resulting beams should have near identical angular distributions, as shown in figure 18. As the velocity distribution becomes wider, we would expect a larger effect, especially along the edge of the main droplet beam: in the initial quasi-linear region of the angular distribution and fixed polar angle, 


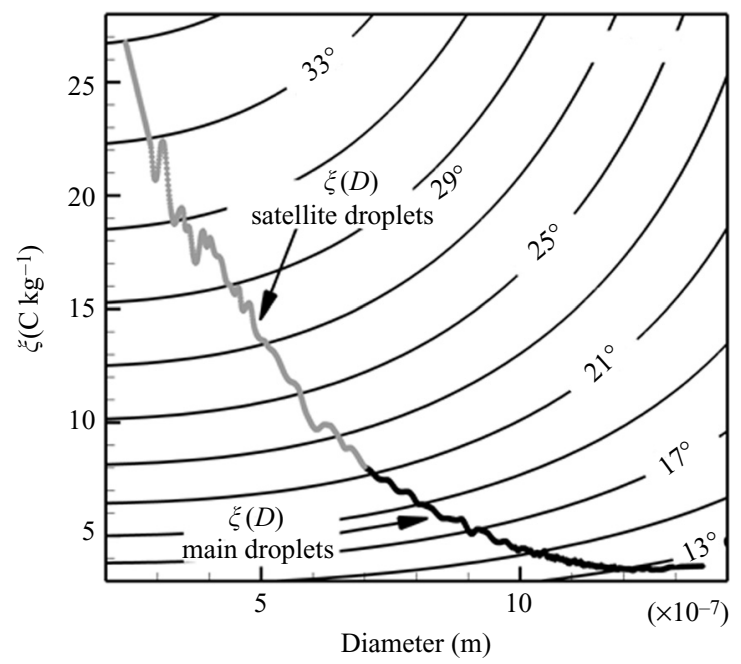

FIGURE 19. Contour plot of the exit angle as a function of the charge to mass ratio $\xi$ and diameter $\left(I_{B}=125 \mathrm{nA}\right)$. The larger $\xi$-component of the gradient of the exit angle is explained by the presence of $\xi$ in the controlling factor of the expression for the exit angle.

the reduction in current caused by the smaller initial velocity of a larger droplet is compensated by the current associated with a smaller droplet with a larger initial velocity; this compensating mechanism disappears at the edge of the beam because of the separation of droplets by diameters, making the edge smoother as the velocity distribution widens.

Figure 19 plots contours of constant exit angle as a function of the charge to mass ratio $\xi$ and diameter $D$ of a droplet. The exit angle is computed for the $125 \mathrm{nA}$ beam, using a value of one for both the strength factor and the dimensionless initial velocity. We also plot the average charge to mass ratio of the $125 \mathrm{nA}$ beam droplets. It is a familiar result of electrostatics that droplets with different charge to mass ratios will have identical trajectories if and only if they have the same initial positions and retarding potentials. For example, in our model and considering $\tilde{v}_{N}=\kappa=1$, droplets with different $\xi$ will have different exit angles because they have different retarding potentials, $\phi_{J}+v_{J}^{2} / 2 \xi$. Further application of this result must be done with caution because although our field is electrostatic, it is a function of the droplet diameter (it also depends on the strength factor, but we are considering $\kappa=1$ ). Thus, droplets with identical $\xi$ and therefore the same retarding potential, but with different diameters, will also have different exit angles, as illustrated by figure 19. The dependence of the exit angle on the charge to mass ratio is due to the presence of $\xi$ in the controlling factor of (23), whereas the effect of the droplet diameter is due to the presence of $\lambda_{o}$ in the droplet size factor. The controlling versus droplet size factor effect is illustrated in figure 19 by the gradient of the exit angle, whose $\xi$-component is clearly larger than its $D$-component throughout the graph. This gradient, together with the $\xi(D)$ path of the electrospray droplets, determines the contributions of $\xi$ and $D$ to the actual range of exit angles.

\subsection{Numerical integration of multiple trajectories using a physical law for the strength parameter $\kappa$}

The analytical solution provides a functional form describing the effect of the beam parameters on its structure. This is possible at the cost of accuracy: although our 
definition of the space charge region boundary yields a good estimate for the exit angle, the analytical solution does not solve the smaller contribution from the external field downstream of the space charge region. Furthermore, the use of $H=\kappa$ is inappropriate for the smaller satellite droplets. Both problems can be addressed by numerically integrating the differential equations of the beam model while using a scheme for computing $H(\kappa)$. We have done this by dividing the experimental diameter distribution $f(D)$ into $m$ segments made of droplets with identical diameters and charge to mass ratios, $D_{i}$ and $\xi_{i}$, and carrying a net current $I_{i}, I_{B}=\sum_{i=1}^{m} I_{i}$, $i=1, \ldots, m$. Each segment is further divided into $n$ beamlets with equal currents, $I_{i j}=I_{i} / n, j=1, \ldots, n$, and with initial velocities $v_{0 i j}$ determined from the assumed normal velocity distribution:

$$
\frac{n}{\sigma_{v} \sqrt{2 \pi}} \int_{v_{0 i j}}^{\infty} \exp \left(-\left(\frac{v-v_{J}}{\sqrt{2} \sigma_{v}}\right)^{2}\right) \mathrm{d} v=j-\frac{1}{2} .
$$

These definitions are incorporated in the equations governing the trajectories of the $m \times n$ beamlets,

$$
\begin{gathered}
v_{i j}^{2} \frac{\mathrm{d}^{2} R_{i j}}{\mathrm{~d} z^{2}}+v_{i j} \frac{\mathrm{d} v_{i j}}{\mathrm{~d} z} \frac{\mathrm{d} R_{i j}}{\mathrm{~d} z} \\
=\frac{\xi_{i} \kappa_{i j} I_{B} \lambda}{2 \pi \varepsilon_{0} v_{J}}\left[\frac{8 R_{i j}}{\left(\left(\lambda+\lambda_{i}\right)^{2}+4 R_{i j}^{2}\right)^{3 / 2}}+\frac{\sqrt{4 R_{i j}^{2}+\left(2 \lambda+\lambda_{i}\right)^{2}}-\left(2 \lambda+\lambda_{i}\right)}{R_{i j} \sqrt{4 R_{i j}^{2}+\left(2 \lambda+\lambda_{i}\right)^{2}}}\right]+\xi_{i} E_{r}^{e x t}, \\
v_{i j} \frac{\mathrm{d} v_{i j}}{\mathrm{~d} z}=\xi_{i} E_{z}^{e x t}, \\
E^{e x t}=-\nabla \phi^{e x t}, \\
\phi^{e x t}\left(z_{0}\right)=\phi_{J}, \quad R_{i j}\left(z_{0}\right)=\frac{j}{n} R_{J}, \quad R_{i j}^{\prime}\left(z_{0}\right)=0, \quad v_{i j}\left(z_{0}\right)=v_{0 i j}, \\
\kappa_{i j}(z)=\frac{\sum I_{k l}, \forall k, l \ni R_{k l}(z) \leqslant R_{i j}(z)}{\sum I_{k l}, \forall k, l \ni R_{k l}(z) \leqslant R_{i n}(z)},
\end{gathered}
$$

$\lambda$ is the breakup wavelength of the average main droplet $(3.10,3.13$ and $2.82 \mu \mathrm{m}$ for the 125,92 and $69 \mathrm{nA}$ beams, respectively), while $\lambda_{i}$ is the wavelength associated with the $i$-segment droplet. The trajectory equations are coupled by the strength factor $\kappa_{i j}$. The strength factor $\kappa_{i j}$ of the $i j$-beamlet is the sum of the currents of beamlets with trajectories falling under $R_{i j}$; the sum is normalized so that $\kappa_{i n}$ is equal to one, i.e. the most external beamlet of every $i$-segment sees the full force of the line of point charges model. $\kappa_{i j}$ is computed at each integration step, and provides a mechanism for the angular separation by droplet diameters observed in the experimental sprays. Before integrating the equations, the external electric field is solved numerically in a geometry containing the perforated extractor electrode and the emitter tip. The latter is a conducting cylinder with the outer radius of the electrospray needle, and which ends in a $45^{\circ}$ cone. The vertex of the cone is located at $z=0$. The initial conditions are placed at the axial position $z_{0}$ where the numerical solution of the potential field is equal to the jet breakup potential; $z_{0}$ is 196,264 and $196 \mu \mathrm{m}$ for the 125,92 and $69 \mathrm{nA}$ beams, respectively, while the axial electric fields at $z_{0}$ are $1.79 \times 10^{6}, 1.25 \times 10^{6}$ and $1.70 \times 10^{6} \mathrm{~V} \mathrm{~m}^{-1}$. 
(a)

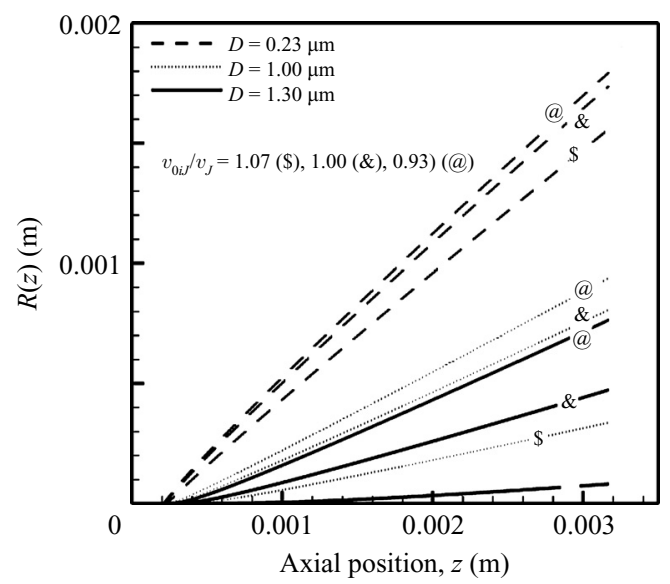

(b)

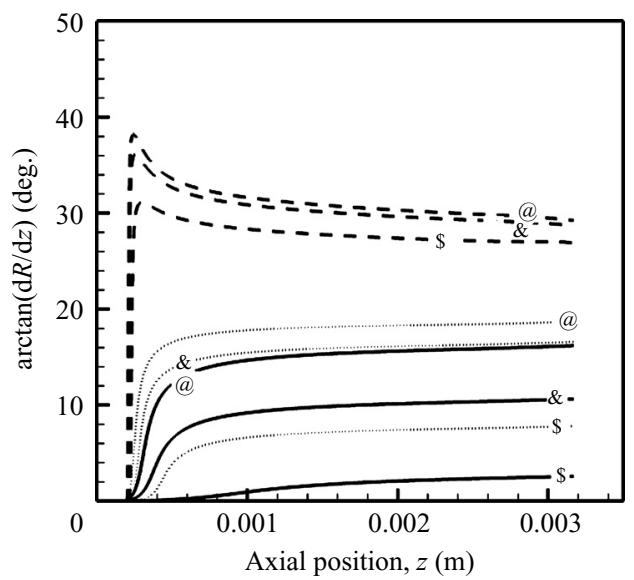

FIGURE 20. Numerical integration of droplet trajectories and inverse tangents $\left(I_{B}=125 \mathrm{nA}\right)$. The curves are for three droplet diameters and three initial velocities.

Figure 20 shows typical trajectories $R_{i j}(z)$ and their inverse tangent $\arctan \left(R_{i j}^{\prime}(z)\right)$ for the $125 \mathrm{nA}$ beam. The diameter distribution has been divided into 175 beamlets $(m=25, n=7)$, and the standard deviation of the initial velocity distribution is $5 \%$ of the jet velocity, $\sigma_{v}=0.05 v_{J}$. The curves in figure 20 are for two main droplet with diameters of 1.30 and $1.00 \mu \mathrm{m}$, and for one satellite droplet, $D=0.23 \mu \mathrm{m}$. The three curves for each droplet have different initial velocities, $v_{0 i j} / v_{J}=1.07,1.00$ and 0.93 , the higher the initial velocity the nearer the trajectory to the $z$-axis. The trajectories resemble straight lines at the scale of the figure. Satellite droplets separate early from the main droplet trajectories, the latter ones remaining closer to the $z$-axis. This is consistent with the strong angular separation observed in experiments. On the other hand, the separation between main droplets of different diameters is much weaker, with most of the two main droplet ranges overlapping each other. The inverse tangent curves display the initial expansion of the beam with greater resolution. The inverse tangent increases rather fast until it reaches a maximum in the case of the satellite droplets, or an asymptotic growth for the main droplets. The inverse tangent of satellite droplets decreases beyond its maximum because at that large radius the space charge force becomes negligible compared to the external electric force, and the motion of the particle is dominated by axial acceleration and the resulting bending of the trajectory towards the axis. The inverse tangent of the main droplets does not exhibit a maximum because although the space charge force becomes increasingly small, it is always comparable to the external electric force and acts against the inward bending of the trajectory associated with the axial acceleration.

Figure 21 shows the angular distributions derived from the numerical solution. They match the experimental profiles better than the analytical solutions in figure 15: the main droplet distributions follow the experimental curves closely, and satellite and main droplets are well separated. The improvement should be expected because the numerical scheme implements the effect of the external field on $R_{i j}^{\prime}(z)$, as well as a mechanism for computing $H\left(\kappa ; D, v_{0}\right)$. The computed satellite distributions do not reproduce the largest angles of the experimental distributions, probably because we did not characterize these most external regions of the beams with the ICD. As a result, we expect that the diameter distributions of figure 10 do not include 
(a)

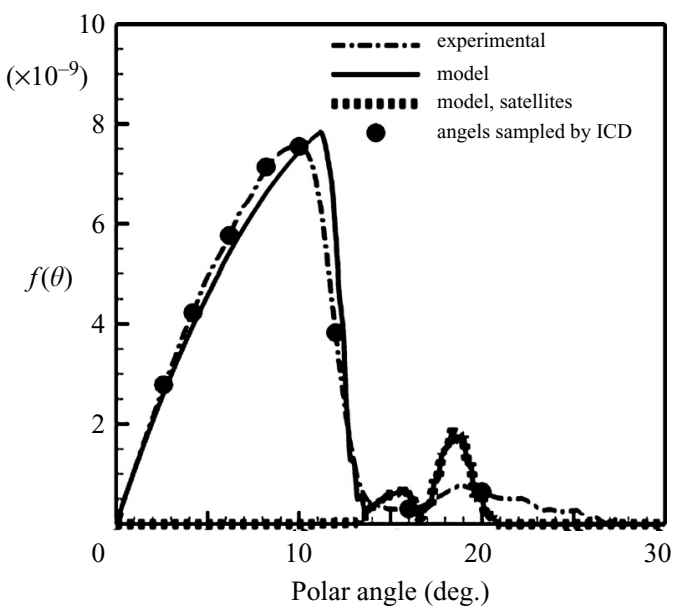

(b)

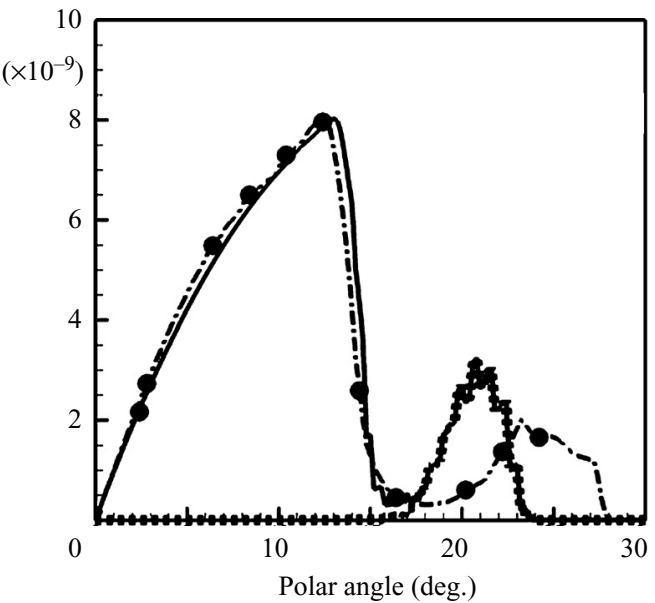

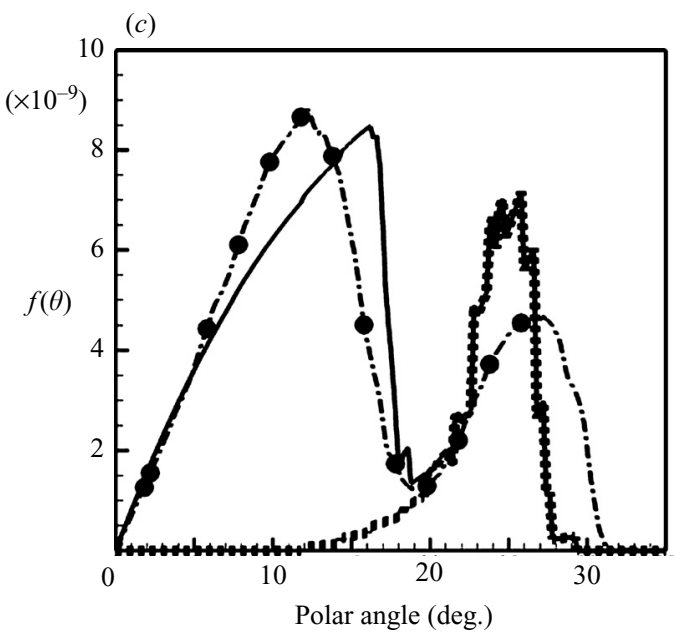

FIGURE 21. Experimental angular profiles and numerical solutions for the three higher beam currents. (a) $I_{B}=69 \mathrm{nA},(b) 92 \mathrm{nA},(c) 125 \mathrm{nA}$. The model predicts well the shape of the distributions and the separation between main and satellite droplets. We also plot the angles for which we have measured the diameter and specific charge of droplets.

the smallest satellite droplets, and therefore we cannot compute the trajectories with largest exit angles. Figure 21 shows the polar angles where the electrosprays were sampled by the ICD: the largest polar angles are $20^{\circ}, 24^{\circ}$ and $26^{\circ}$ for the 69,92 and $125 \mathrm{nA}$ beams, respectively; these angles compare well with the maximum angular range of the computed distributions.

\section{Summary}

We have characterized five electrosprays of a propylene carbonate solution in vacuum. The broadness of these beams increases with the beam current. At the lowest current there is a single conical beam made of main droplets. Satellite droplets, which have smaller diameter and larger charge to mass ratio than main droplets, are generated at larger beam currents and form a second coaxial beam surrounding 
the main droplets. In all cases, the current per solid angle versus polar angle associated with main droplets resembles a rectangle function. We have measured the charge and diameter of individual droplets with the combination of an induction charge detector and a retarding potential analyser, and mapped the distributions of these parameters throughout the polar range of the beams. There is a good mixing of main droplet diameters throughout most of the inner beam, followed by a concentration of smaller main droplets along the inner beam edge. The charge and diameter distributions of either main or satellite droplets are broader than their charge to mass ratio distributions; the charge to mass ratio at fixed diameter is narrowly distributed; and for main droplets, the charge to mass ratio is a weak function of the diameter, becoming nearly constant when satellite droplets are absent from the spray. The latter experimental observation contradicts the applicability of the equipotential electrification assumption to model the capillary breakup of cone-jets.

Our beam model is based on an Eulerian formulation without explicit time dependence, which is made possible by the use of a simplified expression for the space charge electric field. This field is induced by a periodic distribution of the average main droplet along the axis, referred to as line of point charges model. The resulting radial electric field is a function of the radial position, the properties of the average main droplet, and the diameter of the droplet on which the field is acting. The simplified equations of motion reveal the existence of an initial region where: $(a)$ the external electric field does not alter substantially the very large initial axial velocities of the droplets; and $(b)$ the radial velocities of the droplets are driven by the space charge. We have found an analytical solution for the tangent of the droplet trajectory in this initial region which, combined with a criterion for estimating and defining its boundary, yields the angle of the trajectory as it exits the space charge region. Furthermore, for the geometry and potential differences typical of an emitterextractor electrospray source, the tangent of the trajectory changes little beyond the initial region. Therefore the analytical solution reproduces, in terms of beam and droplet parameters, the main features of the beam structure measured downstream of the extractor. In particular, the analytical solution explains: the parabolic relation between accumulated beam current and polar angle (or equivalently the rectangle function aspect of the beam profiles); the existence of an outer beam associated with satellite droplets; and the initial weak angular separation by diameters of main droplets, and why the diameter separation becomes noticeable at the edge of the main droplet beam.

Although the analytical solution is a sound framework for explaining the structure of the sprays, it does not describe the effect of the external electric field beyond the space charge dominated region, nor does it provide a satisfactory way of relating the current distribution to the space charge force. We have solved these shortcomings by computing the external electric field with a numerical package, and integrating the trajectory equations for a small set of droplets representative of the beam. This formulation implements a Coulombic interaction between trajectories with an electric field that has the functional form of the line of point charges model, and whose intensity at $R$ is proportional to the sum of the currents of trajectories between the axis and $R$. The comparison between the numerical solution and measured beam profiles, shown in figure 21 , is fairly good. In particular, we are able to reproduce the near complete angular separation between main and satellite droplets. Such a good agreement is interesting because of the intrinsic differences between the physics of the actual beam and the model (i.e. Coulombic time-dependent interaction 
between individual droplets versus a steady-state Eulerain model of trajectories of representative droplets), and the strong dependence of the model results on the initial velocity of the droplets (errors made on the measurement of the initial velocity amplify the difference between model and experimental results). We think that the latter finding validates our estimates of the jet velocity and potential computed via time of flight and retarding potential analysis. Conversely, we could use the beam model combined with the simpler beam profile measurement to estimate the jet velocity and potential, i.e. we could find the initial and boundary conditions of the model that reproduce a good match between the experimental beam profile and the model solution. This result would simplify the determination of key parameters required for the study and modelling of capillary breakup of charged jets.

I am indebted to Dr I. Katz for suggesting that the line of point charges model could be used to estimate the space charge electric field. This research was largely motivated by the interaction with Professor J. Fernández de la Mora. The research described in this paper was carried out at the Jet Propulsion Laboratory, California Institute of Technology, Pasadena, CA 91108, USA, under a contract with the National Aeronautics and Space Administration.

\section{REFERENCES}

Ashgriz, N. \& Mashayek, F. 1995 Temporal analysis of capillary jet breakup. J. Fluid Mech. 291, $163-190$

Chandrasekhar, S. 1981 Hydrodynamic and Hydromagnetic Stability, pp. 515-576. Dover.

Cloupeau, M. \& Prunet-Foch, B. 1989 Electrostatic spraying of liquids in cone-jet mode. J. Electrostat 22, 135-59.

Collins, R. T., Harris, M. T. \& Basaran, O. A. 2007 Breakup of electrified jets. J. Fluid Mech. 588, $75-129$.

Enloe, C. L. \& Shell, J. R. 1992 Optimizing the energy resolution of planar retarding potential analyzers. Rev. Sci. Instrum. 63, 1788-1791.

Fenn, J. B., Mann, M., Meng, C. K., Wong, S. K. \& Whitehouse, C. M. 1989 Electrospray ionization for mass spectrometry of large biomolecules. Science 246, 64-71.

Fernández DE LA MoRA, J. 1992 The effect of charge emission from electrified liquid cones. J. Fluid Mech. 243, 561-574.

Fernández, De la Mora, J. 2007 The fluid dynamics of Taylor cones. Annu. Rev. Fluid Mech. 39, 217-243.

Fernández de la Mora, J. \& Loscertales, I. G. 1994 The current transmitted through an electrified conical meniscus. J. Fluid Mech. 260, 155-184.

Gamero-Castaño, M. \& Hruby, V. 2002 Electric measurements of charged sprays emitted by cone-jets. J. Fluid Mech. 459, 245-276.

Gamero-Castaño, M. 2002 Electric-field-induced ion evaporation from dielectric liquid. Phys. Rev. Lett. 89, 147602.

Gamero-CastaÑo, M. 2007 Induction charge detector with multiple sensing stages. Rev. Sci. Instrum. 78, 043301.

GaÑán-Calvo, A. M. 1997 Cone-jet analytical extension of Taylor's electrostatic solution and the asymptotic universal scaling laws in electrospraying. Phys. Rev. Lett. 79, 217.

GaÑán-CALVO, A. M. 1998 Generation of steady liquid microthreads and micron-sized monodisperse sprays in gas streams. Phys. Rev. Lett. 80, 285-288.

López-Herrera, J. M. \& Gañán-Calvo, A. M. 2004 A note on charged capillary jet breakup of conducting liquids: experimental validation of a viscous one-dimensional model. J. Fluid Mech. 501, 303-326.

Martínez-SÁNChEZ, M. 2004 16.522 Space Propulsion. Massachusetts Institute of Technology OpenCourseWare. http://ocw.mit.edu/OcwWeb/Aeronautics-and-Astronautics/16522Spring2004/CourseHome/index.htm. 
McEwen, A. B., NGo, H. L., LeCompte, K. \& Goldman, J. L. 1999 Electrochemical properties of imidazolium salt electrolytes for electrochemical capacitor applications. J. Electrochem. Soc. 146, 1687-1695.

Morse, P. M. \& Feshbach, H. 1953 Methods of Theoretical Physics, Part II, p. 1284. McGraw-Hill.

Reiser, M. 1994 Theory and Design of Charged Particle Beams p. 191. John Wiley.

Riddick, J. A, Bunger, W. B. \& Sakano, T. K. 1986 Organic Solvents. Physical Properties and Methods of Purification, 4th edn. Wiley.

Shelton, H., Hendricks, C. D. \& Wuerker, R. F. 1960 Electrostatic acceleration of microparticles to hypervelocities. J. Appl. Phys. 31, 1243-1246.

TAYlor, G. I. 1964 Disintegration of water drops in an electric field. Proc. R. Soc. Lond. A 280, 383-397. 